\title{
Abstracts of the 12th BOMSS Annual Scientific Meeting
}

\author{
P1 - Efficacy of pre-operative 'liver shrinking' dietary regimens: a \\ UK national cohort study \\ Sally Abbott ${ }^{1}$, Dr Charlotte Price ${ }^{1}$, Dr Karen Coulman ${ }^{2}$ \\ ${ }^{1}$ Coventry University, Coventry, United Kingdom, ${ }^{2}$ Bristol University, \\ Bristol, United Kingdom
}

Background: Low energy diets (LEDs) $(1000-1200 \mathrm{kcal})$ and very low energy diets (VLEDs) $(<800 \mathrm{kcal})$ have been shown to significantly reduce liver size in preparation for laparoscopic bariatric surgery. However, pre-operative dietary practices vary considerably and research to date has not examined the efficacy of a range of preoperative diets within clinical practice. Thus, our study aimed to establish the variation in, and efficacy of, pre-operative dietary regimes utilised in practice across the UK.

Methods: We conducted a retrospective cohort study of all patients who underwent a primary bariatric procedure at 16 participating UK bariatric centres between 1st January 2019 to 31st December 2019. Patient demographic data (gender, age, ethnicity), diabetes status and weight at baseline and on the day of surgery was collected. We also collected data about the pre-operative diet regimes, including kcals/day and duration. A multilevel linear random effects model was developed to examine predictors of weight on day of surgery. Baseline weight, diabetes status, diet duration and kcals/day were included in the model.

Results: A total of 2,092 patients were included in the analysis. Diets ranged from $<800 \mathrm{kcal}$ to $>1200 \mathrm{kcals} /$ day and lasted one week to $\geq 5$ weeks. The most common diets followed by patients were 800 $1000 \mathrm{kcals}(55.7 \%)$ and lasted 2 weeks $(71.4 \%)$. The unadjusted mean weight loss across all patients was $5.43 \mathrm{~kg}$ (95\% CI 5.18 to 5.68). Weight on the day of surgery was, on average, $0.79 \mathrm{~kg}$ less for patients with diabetes compared to those without diabetes $(\mathrm{p}=0.002)$, and also less for 4 week $(1.90 \mathrm{~kg}, \mathrm{p}=0.035)$ and $\geq 5$ week diets $(3.54 \mathrm{~kg}, \mathrm{p}=0.001)$, compared to a 1 week diet. Patients on a $<800 \mathrm{kcals} /$ day diet were, on average, $8.86 \mathrm{~kg}$ lighter than $>1200$ $\mathrm{Kcals} /$ day diets on the day of surgery $(\mathrm{p}=0.016)$. There was no significant difference in weight between VLED of $<800 \mathrm{kcal} /$ day diets and LEDs of either $800-1000 \mathrm{kcal} /$ day $(\mathrm{p}=0.194)$ or $1000-1200 \mathrm{kcal} /$ day $(\mathrm{p}=0.212)$.

Conclusions: A range of pre-operative diets are used in clinical practice in the UK, reflecting a need for standardised guidance in this area. Our study found that lower kcal and longer duration pre-operative diets were associated with greater pre-operative weight loss. Further research is required to confirm our findings that patients with diabetes respond better to preoperative diets and to establish the adequate level of weight loss as proxy for liver shrinkage prior to bariatric surgery.

P2 - Using the Ensuring Quality Information for Patients Tool to Assess Patient Information on Sleeve Gastrectomy Websites

Dr Husham Ahmad ${ }_{-}$, Mr Tanvir Hossain ${ }^{2}$, Mr Naveed Hossain ${ }^{3}$
${ }^{1}$ Nottingham University Hospitals, Nottingham, United Kingdom, ${ }^{2}$ Nottingham University Hospitals, Nottingham, United Kingdom, ${ }^{3}$ Whittington Hospital, London, United Kingdom

Background: Sleeve gastrectomy is the most common bariatric procedure world-wide. Quality patient information resources can aide consent, expectations and compliance with post-operative plans. Online resources are likely the first port of call for patients, awareness of their standard can guide new patient information media.

Objective: The objective of this study was to identify the quality of information available to general members of the public regarding sleeve gastrectomy. Methods: A search was conducted using the term 'sleeve gastrectomy' into Google's search engine and the top 50 highest ranking results were identified. These were assessed using the validated Ensuring Quality Information for Patients (EQIP) tool.

Results: The 50 highest ranking websites that give patient information on sleeve gastrectomy were analysed. These were amongst the first 83 results, 23 were unrelated to patient information. The overall median EQIP score was $63.2 \%$ (IQR 15.8$)$, mean score $65.5 \%( \pm 11.34)$. Of these 50 , $10 \%(5 / 50)$ were NHS trusts, $64 \%$ (32/50) were private hospitals and $26 \%$ $(13 / 50)$ were medical education websites. The mean scores for each were $74.4 \%, 62.8 \%$ and $68.7 \%$ respectively.

Conclusions: The assessment of the quality and reliability of online resources indicate that there are higher scores for information from NHS trusts than independent ones.

P3 - Comparing health-related quality of life outcomes in women of reproductive age following Sleeve Gastrectomy vs Roux en y Gastric Bypass Procedure.

Miss Kiran Akmal ${ }^{1}$, Dr Stephanie Attersley-Smith ${ }^{2}$, Dr Kathryn Hart ${ }^{3}$, Dr Martin Whyte ${ }^{3}$, Professor Jill Shawe ${ }^{3}$, Dr Sophia Stone ${ }^{2}$

${ }^{1}$ Brighton and Sussex Medical School, East Sussex, United Kingdom, ${ }^{2}$ St Richard's Hospital, West Sussex, United Kingdom, ${ }^{3}$ University of Surrey, Guildford, United Kingdom

Women of reproductive age account for 53-80\% of all women undergoing bariatric surgery ${ }^{1}$. Literature investigating the impact of bariatric surgery on health-related quality-of-life (HRQoL) within this specific demographic is minimal ${ }^{2}$. This is the first study to exclusively investigate the impact of different bariatric procedures on HRQoL and weight loss outcomes in women under the age of $45^{3}$.

A prospective cohort study was conducted between 2018-2021. HRQoL outcomes for participants undergoing Sleeve Gastrectomy (SG) or Rouxen-y Gastric Bypass (RYGB) were assessed using the bariatric obesity specific survey at four intervals over 12-months. Descriptive data analysis was conducted to observe general trends and a one-way ANOVA was performed to assess significance.

HRQoL outcomes increased following bariatric surgery irrespective of procedure type $(\mathrm{p}<0.01)$. However, no statistically significant difference was identified between RYGB or SG for both HRQoL and weight loss 
outcomes ( $>0.05)$ (Figure 1). Inter-domain analysis showed a decrease in sexual function for RYGB ( $p>0.05$ ) at 6-and 12-month follow-up.

Bariatric surgery in women of reproductive age is associated with an increase in HRQoL. Our comparison between the most performed bariatric procedures identified minimal difference in HRQoL and weight loss outcomes. These findings are vital for informing patient decision-making when considering bariatric procedure type.

\section{COMPARISION OF HRQOL AND WEIGHT LOSS OUTCOMES BETWEEN RYGB AND SG PROCEDURE}

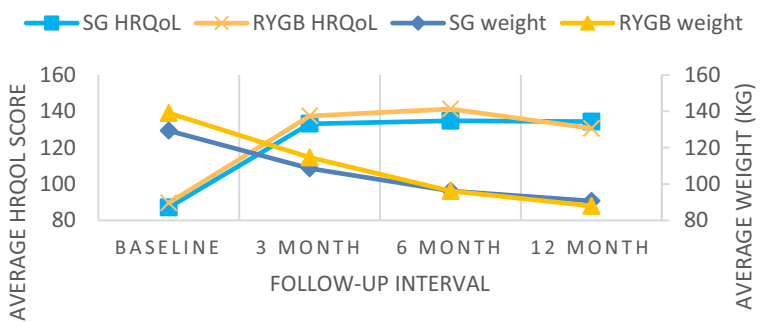

Figure 1: A graph comparing the average HRQoL outcomes and weight (kg) for participants who underwent RYGB vs SG over the 12-month follow-up period. The graph shows there was no statistically significant difference between HRQoL scores for SG and RYGB ( $p>0.05)$ as well as no statistically significant difference in weight loss between the two procedures $(p>0.05)$.

\section{References:}

1 Vieira A, Santa Cruz F, Júnior A, Ferraz Á, Kreimer F. Evaluation of the Quality of Life in Women of Reproductive Age After Bariatric Surgery. Bariatric Surgical Practice and Patient Care. 2020; 15. doi: 10.1089/ bari.2019.0051.

2 Cooiman MI, Aarts EO, Janssen IMC, Hazebroek EJ, Berends FJ. Weight Loss, Remission of Comorbidities, and Quality of Life After Bariatric Surgery in Young Adult Patients. Obes Surg. 2019 Jun;29(6):1851-1857. doi: 10.1007/s11695-019-03781-z.

3 Guy P. Childbearing for women born in different years, England and Wales: 2017. London: Office for national statistics; 2019

P4 - Emergency conversion of single anastomosis gastric bypass (SAGB) to Roux-en-y Gastric bypass (RYGB) due to afferent loop obstruction (ALO), an uncommon complication post SAGB. Case report and review of literature

$$
\begin{aligned}
& \text { Mr Mahmoud Al-ardah_, Mr Ian Finlay }{ }^{1} \\
& { }^{1} \text { Royal Cornwall Hospital, Truro, United Kingdom }
\end{aligned}
$$

Introduction: Afferent loop syndrome or obstruction is an infrequent mechanical complication after gastric surgery and gastrojejunostomy. It can be either acute or chronic. In the acute type favourable outcome is correlated with an expedient diagnosis and early surgical intervention. We present a case of (ALS) post single anastomosis gastric bypass (SAGB). Case presentation: A 49-year- old male patient presented to our Bariatric Unit through the ED with severe progressive upper abdominal pain for the last 12 hours, associated with significant sickness and decrease in oral intake.

He had a SAGB for obesity 2 years ago performed elsewhere, with endoscopic dilatation of the anastomosis performed twice before.

CT scan abdomen and pelvis showed small bowel obstruction with a transition point at the gastrojejunostomy causing severe dilatation of the small bowel and stomach remnant. A laparoscopic to open revision of the SAGB to a Roux en Y gastric bypass was performed. The patient made an eventful recovery and was discharged home 10 days post operatively.
Conclusion: Afferent loop obstruction is rare complication of SAGB for obesity and it should considered as be part of the working diagnosis in patients presenting with obstructive symptoms. Prompt diagnosis and surgical intervention is crucial.

P5 - Safety of bariatric surgery during COVID-19 pandemic, is there a need to screen low-risk patients?

Dr Emad Aljohani ${ }^{1}$

${ }^{1}$ Prince Sattam Bin Abdullaziz University, Riyadh, Saudi Arabia

Background: There seems to be a consensus in the current published literature on postponing elective, non-urgent surgery on COVID-19positive patients. However, so far, no recommendations have been published on when and how to start carrying out elective, non-urgent surgery on COVID-19-negative patients after the epidemic peak.

Methodology: A retrospective chart review of patients who underwent bariatric surgery between March to June 2020, during the pandemic of COVID 19. The study participants were male and females with a BMI > 30 with a respiratory score of $\leq 3$.

Results: The mean age of the patients was $32.73 \pm 7.81$ years, and a higher proportion $(n=48 ; 56.5 \%)$ of them were males. Only $(n=1 ; 1.1 \%)$ of the patient was tested for Covid19 by RT-PCR before surgery and tested negative. Post-surgery, none of the patients developed any complications, and none of them were admitted to the ICU.

Conclusion: During the COVID-19 pandemic, before considering patients for elective surgery, they should be screened. For patients who are obese or have underlying comorbidities, if on screening their respiratory score is $\leq 3$ indicating a low risk of respiratory illness, elective procedures should continue. Strict precautionary measures should be followed, and a limited number of surgeries should be performed.

Keywords: COVID-19, Bariatric surgery, respiratory score, low risk, elective surgery

P8 - Laparoscopic Roux-en-Y gastric bypass versus Sleeve gastrectomy for dyslipidemia - a comparison of 2,059 dyslipidemic patients

Mr Chanpreet Arhi ${ }_{-}^{1}$, Mr Md Tanveer Adil ${ }^{1}$, Dr Minil Patel ${ }^{1}$, Dr Anil Rai ${ }^{1}$, Mr Tanmoy Mukherjee ${ }^{1}$, Mr Khanzadi Chandio ${ }^{1}$, Nissy Pius ${ }^{1}$, Ms Carol Villani $^{1}$, Mr Aruna Munasinghe ${ }^{1}$, Mr Farhan Rashid ${ }^{1}$, Mr Douglas Whitelaw $^{1}$, Mr Vigyan Jain ${ }^{1}$, Mr Periyathambi Jambulingam ${ }^{1}$, Mr Omer Al-Taan ${ }^{1}$

${ }^{1}$ Luton And Dunstable Hospital, , United Kingdom

Introduction: Published randomised controlled trials (RCTs) have failed to show a statistically significant advantage of laparoscopic Roux-en-Y gastric bypass (LRYGB) over laparoscopic sleeve gastrectomy (LSG) in the improvement of dyslipidaemia due to small subgroup sizes.By performing an analysis on a larger sample size, our aim was to determine which procedure is likely to produce the greatest benefit for dyslipidaemia.

Method: A comprehensive database search was undertaken for observational (OS) and RCTs published until 31st December 2020 comparing LRYGB with LSG. The primary outcome was rate of resolution and/or partial improvement of dyslipidemia at least 12 months after surgery.Data from our institute of 106 patients were also included. The magnitude of the experimental effect was calculated in terms of odds ratio (OR) while accounting for co-morbidities, gender, age and BMI.

Results: Inclusion of 1148 LRYGB and 911 LSG patients from 7 RCTs and 18 OS demonstrated the primary outcome was achieved in $73 \%$ LRYGB vs $50.7 \%$ LSG(p<0.0001) with OR 2.29(95\%CI $1.69-3.10$ $\mathrm{p}<0.00001)$ for LRYGB compared with LSG. Separate analysis of only 
RCTs (OR 2.28;95\%CI 1.21-4.29; $\mathrm{p}=0.01)$ or only OS (OR 2.25; 95\%CI 1.56-3.24; $\mathrm{p}<0.0001)$ generated similar findings.

Conclusion: LRYGB achieves greater improvement in dyslipidaemia compared to LSG at more than 12 months after bariatric surgery.

P9 - Safety of bariatric surgery during the Covid-19 pandemic - a retrospective cohort study from a UK unit

Mr Chanpreet Arhi ${ }^{\mathbf{1}}$, Dr Osama Alhabsha ${ }^{1}$, Mr Ravikrishna Mamidanna ${ }^{1}$, Mr Aruna Munasinghe ${ }^{1}$, Mr Farhan Rashid ${ }^{1}$, Mr Periyathambi Jambulingam $^{1}$, Mr Douglas Whitelaw ${ }^{1}$, Mr Vigyan Jain ${ }^{1}$, Mr Omer AlTaan $^{1}$, Mr Md Tanveer Adil ${ }^{1}$

${ }^{1}$ Luton And Dunstable Hospital, , United Kingdom

Background: The strain on resources during the pandemic has meant bariatric surgery has been delayed. To mitigate against further disruption, we established a 'green' pathway. The aim of this study was to compare the 30-day complications and length of stay(LOS) during the pandemic, with patients operated one year previously.

Methods: The first 100 consecutive bariatric procedures from July 2020 onwards were compared with 100 consecutive patients from July 2019 for complications (Clavien-Dindo $\geq$ II) and LOS using both univariate and multivariate analysis, with the latter accounting for gender, pre-op BMI, type of procedure and comorbidities. Two week isolation and pre-operative PCR were mandatory in 2020, with no pre-determined BMI or co-morbidity precluding surgery.

Results: In 2020, 67 LRYGB, 32 LSG and 1 DS were carried out compared with 79, 21 and 0 respectively in $2019(\mathrm{p}=0.12)$. A similar proportion were revisional ( 8 in 2019 vs 10 in 2020). There was no difference in mean pre-operative BMI ( 46.4 in 2020 vs $48.6 \mathrm{~kg} / \mathrm{m} 2 \mathrm{p}=0.17$ ), females or co-morbidities (Type II DM, GERD, hypertension, OSA, or hyperlipidaemia) between the two years. Although median LOS was 1 day for both groups, a significantly higher proportion stayed for 2 days in 2019 (16 vs 9 $\mathrm{p}=0.04$ ). There was no significant increase in the risk of a complications in 2020 (OR 0.66 CI 0.12-3.70 p=0.64). There were no positive Covid cases post-operatively.

Conclusion: This study demonstrates bariatric surgery is safe through the Covid pandemic with the appropriate precautions.

\section{P10 - The changing face of bariatric surgery: experience of a UK unit}

Mr Chanpreet Arhi ${ }^{1}$, Mr Periyathambi Jambulingam ${ }^{1}$, Mr Aruna Munasinghe $^{1}$, Mr M $\bar{d}$ Tanveer Adil ${ }^{1}$, Dr Sharukh Zuberi ${ }^{1}$, Mr Farhan Rashid $^{1}$, Mr Douglas Whitelaw ${ }^{1}$, Mr Vigyan Jain ${ }^{1}$, Mr Omer Al-Taan ${ }^{1}$

${ }^{1}$ Luton And Dunstable Hospital, , United Kingdom

Background: The availability of metabolic \& bariatric surgery (MBS) is poor in the UK, particularly for ethnic minorities. There is also growing evidence that MBS should be considered for lower BMI to prevent future co-morbidities. The aim of this study was to determine whether MBS provision reflects these considerations.

Method: Retrospective analysis of primary NHS procedures between 2012 to 2019 recorded in the NBSR database, split into biennial groups. Age, BMI, weight, co-morbidities and type of procedure were compared between year groups using Mantel-Haenszel test of trend for categorical data, and Kruskal-Wallis for continuous data.

Results: 1725 patients were included, with LRYGB being most common. Proportion of LSG peaked in 2016/17(38.1\%), followed by a fall to $29.2 \%$ in $2018 / 19(\mathrm{p}<0.001)$.

There was a gradual significant reduction from $2012 / 13$ to $2018 / 19$ in Type II DM(43.8\% to $32.5 \%$ ), Hypertension $(49.3 \%$ to $38.7 \%$ ), $\operatorname{OSA}(52.1 \%$ to $29.7 \%)$ and dyslipidaemia( $27.2 \%$ to $9.3 \%)$, with a fall in median BMI (48.4 to $45.4 \mathrm{p}<0.001)$ and median weight $(136 \mathrm{~kg}$ to $126 \mathrm{~kg}$ $\mathrm{p}<0.001$ ) before surgery. Mobility ( $\geq 3$ flights of stairs: $30.7 \%$ to $47.6 \%$ ) and the proportion from ethnic minorities $(7.7 \%$ to $9.7 \%$ ) increased between $2012 / 13$ to $2018 / 19(\mathrm{p}<0.05)$. Median age remained constant at $47(\mathrm{p}=0.61)$.

Conclusion: The change in co-morbidities, BMI and ethnicity may reflect the use of MBS as an early intervention rather than only as a treatment for established obesity related issues.

P11 - Are we offering patients with obesity Roux-en-Y bypass surgery when they present for anti-reflux surgery? A retrospective hospitalbased cohort study

Tom Arkle ${ }^{\mathbf{1 , 2}}$, Mr Dimitri Pournaras ${ }^{3}$, Mr Stephen Lam ${ }^{1,2}$, Mr Bhaskar Kumar $^{1,2}$

${ }^{I}$ Norwich Medical School, University Of East Anglia, Norwich, United Kingdom, ${ }^{2}$ Norfolk and Norwich University Hospital NHS Trust, Norwich, United Kingdom, ${ }^{3}$ Southmead Hospital, North Bristol NHS Trust, Bristol, United Kingdom

Introduction: In eligible people with obesity, Roux-en-Y gastric bypass (RYGB) has been shown to be highly successful in controlling both GORD symptoms and achieving significant weight loss maintenance, but such an option is underutilised in nonbariatric centres. The aim of our study was to determine the proportion of patients with obesity who may have been eligible for referral for bariatric surgery but instead underwent fundoplication at a non-bariatric centre.

Methods: Using routinely collected hospital data, all patients who underwent fundoplication for GORD at a single academic teaching hospital over a 9-year period were identified and preoperative variables including Body Mass Index (BMI) and co-morbidities were collected.

Results: A total of 321 patients underwent fundoplication between January 2011 and December 2020 of which 133 (41.4\%) had available BMI data and were included in the analyses. BMI $>30 \mathrm{~kg} / \mathrm{m}^{2}$ was seen in 40 patients $(30 \%)$ and $\mathrm{BMI}>35 \mathrm{~kg} / \mathrm{m}^{2}$ in 7 patients $(5.3 \%)$. Of the 7 patients with BMI $>35 \mathrm{~kg} / \mathrm{m}^{2}$, five $(4 \%)$ were eligible, due to obesity associated disease, for bariatric surgery referral.

Conclusions: Given the well-established efficacy of RYGB not only for weight loss maintenance but also as an anti-reflux procedure, we recommend that this highly effective option be more available as a referral option.

\section{References:}

Suter, M., 2020. Gastroesophageal Reflux Disease, Obesity, and Roux-en-Y Gastric Bypass: Complex Relationship-a Narrative Review. Obesity Surgery, 30(8), pp.3178-3187.

Baker, C., 2021. Obesity Statistics. [online] House of Commons Library. Available at: <https://commonslibrary.parliament.uk/research-briefings/ sn03336/> [Accessed 3 May 2021].

NICE, 2017. Complications | Background information | Obesity $\mid$ CKS $\mid$ NICE. [online] Cks.nice.org.uk. Available at: <https://cks.nice.org.uk/ topics/obesity/background-information/complications/> [Accessed 3 May 2021].

Dyer, N. and Pridie, R., 1968. Incidence of hiatus hernia in asymptomatic subjects. Gut, [online] 9(6), pp.696-699. Available at: <https:// gut.bmj.com/content/gutjn1/9/6/696.full.pdf> [Accessed 3 May 2021].

Ortenzi, M., Balla, A., Fontana, G., Marinucci, F., Reggiani, A., Capomagi, P., Bailetti, B., Lezoche, G. and Guerrieri, M., 2020. Factors influencing recurrence after minimally invasive treatment of hiatal hernia - a single center experience. Laparoscopic Surgery, [online] 4, pp.39-39. 
Perez, A., Moncure, A. and Rattner, D., 2001. Obesity adversely affects the outcome of antireflux operations. Surgical Endoscopy, 15(9), pp.986-989.

Kellogg, T., Andrade, R., Maddaus, M., Slusarek, B., Buckwald, H. and Ikramuddin, S., 2006. Anatomic findings and outcomes after antireflux procedures in morbidly obese patients undergoing laparoscopic conversion to Roux-en-Y gastric bypass. Surgery for Obesity and Related Diseases, 3(1), pp.52-57.

Smith, S., Edwards, C. and Goodman, G., 1997. Symptomatic and clinical improvement in morbidly obese patients with gastroesophageal reflux disease following Roux-en-Y gastric bypass. Obes Surg., 7(6), pp.479-84.

NICE, 2014. Recommendations | Obesity: identification, assessment and management | Guidance | NICE. [online] Nice.org.uk. Available at: <https://www.nice.org.uk/guidance/cg 189/chapter/1recommendations\#surgical-interventions $>$ [Accessed 3 May 2021].

\section{P14 - Discharge six: post-operative prescribing and readmission rates} following bariatric surgery

Dr James Bloomfield_ ${ }^{\mathbf{1}}$, Dr Nagarjun Konda ${ }^{1,2}$, Dr Pavandeep Gill ${ }^{1}$, Miss Louise Mitchell ${ }^{1}$, Mrs Jenny Abraham ${ }^{1}$, Mr Vinod Menon ${ }^{1,2}$

${ }^{1}$ Department of Upper GI Surgery, University Hospitals Coventry and Warwickshire NHS Trust, Coventry, United Kingdom, ${ }^{2}$ Warwick Medical School, University of Warwick, Coventry, United Kingdom

Introduction: Complications of Bariatric and Metabolic Surgery (BMS) include bleeding, venous thromboembolism, reflux and anastomotic leaks $(1,2)$. Developed by the bariatric team at a designated BMS centre, the 'discharge six' is a discharge package designed to standardise postoperative protocol, thereby reducing complications and readmissions. This consists of enoxaparin, thrombo-embolus deterrent stockings (TEDs), analgesia, antiemetics, laxatives and a proton-pump inhibitor (PPI).

Methods: Medical records from 100 consecutive patients undergoing BMS in 2019 were analysed, comparing discharge prescribing against the standard of the 'discharge six', and capturing any readmissions up until March 2021.

Results: $84 \%$ of patients were prescribed five of the 'discharge six' and $99 \%$ were prescribed four of the six. The most commonly omitted item was TEDs. There was a $9 \%$ readmission rate for conditions relating to surgery and anaesthesia. The most common cause was constipation (3\%) followed by nausea and vomiting (2\%). Postoperative mortality was $0 \%$ with a $1 \%$ incidence of serious complications. In patients undergoing primary surgery the incidence of serious complications was $0 \%$. When all six items were prescribed only one patient required readmission.

Conclusion: The authors believe these preliminary findings may support the use of the 'discharge six' to standardise postoperative prescribing following BMS.

\section{References:}

1. Lim R, Beekley A, Johnson DC, Davis KA. Early and late complications of bariatric operation. Trauma Surg Acute Care Open. 2018 Oct 9;3(1):e000219. doi: 10.1136/tsaco-2018-000219. PMID: 30402562; PMCID: PMC6203132.

2. Monkhouse SJ, Morgan JD, Norton SA. Complications of bariatric surgery: presentation and emergency management-a review. Ann R
Coll Surg Engl. 2009 May;91(4):280-6. doi: 10.1308/ 003588409X392072. Epub 2009 Apr 2. PMID: 19344551; PMCID: PMC2749388

P15 - Medical Weight Management (Tier 3) Services are not Standardised Across the United Kingdom: Results of a National Survey

Miss Maria Boland $\mathbf{1}_{\mathbf{1}}$, Miss Kerry Aitken ${ }^{2,3}$, Miss Gillian Drummond ${ }^{1,3}$, Mr Brian Joyce ${ }^{1,3}, \overline{\mathrm{Mr}}$ Andrew de Beaux ${ }^{1,3}$, Mr Bruce Tulloh $^{1,3}$, Mr Peter Lamb $^{1,3}$, Mr Andrew Robertson ${ }^{2,3}$

${ }^{1}$ Royal Infirmary Edinburgh, Edinburgh, United Kingdom, ${ }^{2}$ Victoria Hospital Kirkcaldy, Kirkcaldy, Fife, United Kingdom, ${ }^{3}$ South East Scotland Bariatric Service, South East Scotland, United Kingdom

Introduction: Medical weight management (MWM) services (Tier 3) are important as a primary treatment option for obesity and for preparation for bariatric surgery. The aim of this study was to explore the composition of tier 3 services and ascertain opinions of bariatric healthcare professionals on their provision in the UK.

Methods: A literature review was performed and national guidelines for MWM services were reviewed by a multidisciplinary team to develop an online questionnaire survey which was distributed to bariatric professionals electronically.

Results: 66 participants responded, representing $\geq 37$ bariatric units. $94 \%$ $(62 / 66)$ of responders had a Tier 3 service. Variation was seen with regards to MWM MDT make up, referral criteria for surgery, weight loss targets, and duration spent in MWM. $43 \%$ of responders had multiple MWM services and $77 \%$ reported a lack of standardisation.

98\% felt MWM programmes across the UK were not standardised. 46/66 respondents had suggestions to improve MWM services due to a lack of understanding about obesity as a disease and needing broader end points of MWM programmes.

Conclusion: Variation continues to exist in MWM services despite updated guidelines. Health care professionals feel there is a need for improvement. Standardisation of MWM services is required.

P16 - Early post-operative outcomes for patients on immunosuppression undergoing primary bariatric surgery

Ms Caroline Brogan ${ }_{-}$, Ms Emma Kearns ${ }^{2}$, Ms Naomi Fearon ${ }^{2}$, Mr Kin Cheung $\mathrm{Ng}^{2}$, Professor Helen Heneghan ${ }^{1,2}$

${ }^{1}$ UCD School of Medicine and Medical Science, Belfield, Ireland, ${ }^{2}$ Department of Surgery, St. Vincents University Hospital, , Ireland

Background: There is a paucity of data regarding postoperative morbidity and mortality in patients on immunosuppression undergoing bariatric surgery.

Aim: The primary aim was to evaluate 30-day morbidity and mortality in immunosuppressed patients undergoing bariatric surgery. Secondary aims included adherence to an enhanced recovery after bariatric surgery (ERABS) protocol and weight loss outcomes.

Methods: A prospectively maintained database of patients undergoing bariatric surgery between August 2017-February 2020 was used to 
identify patients who were taking immunosuppressant medications preoperatively. Their clinical data were compared to patients not taking immunosuppressants.

Results: Over a 30-month period, 183 patients underwent primary bariatric surgery. Ten patients $(5.5 \%)$ were taking immunosuppressant medication. Mean age and preoperative weight was similar between groups (Table 1). There was no 30-day mortality and overall morbidity was $10.9 \%$. There was no significant difference in 30-day morbidity between groups ( $\mathrm{p}=0.91$, Image 1). Both groups adhered to an ERABS protocol with median length of stay of 2 days in each group. Short-term postoperative weight loss was comparable in both groups (Figure 1).

Conclusion: This small series suggests that patients on immunosuppression are not at increased risk of morbidity or mortality after bariatric surgery, and have comparable weight loss outcomes.

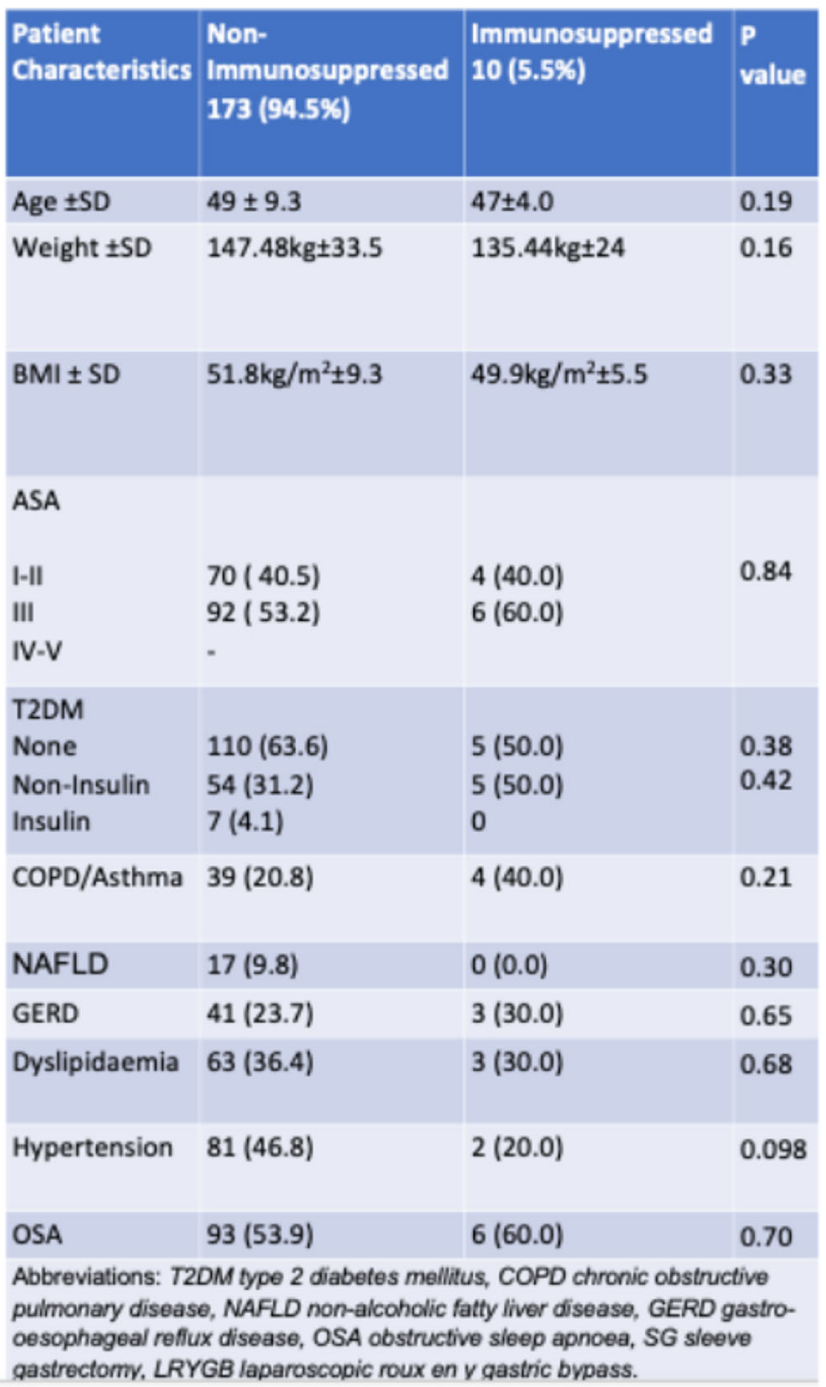

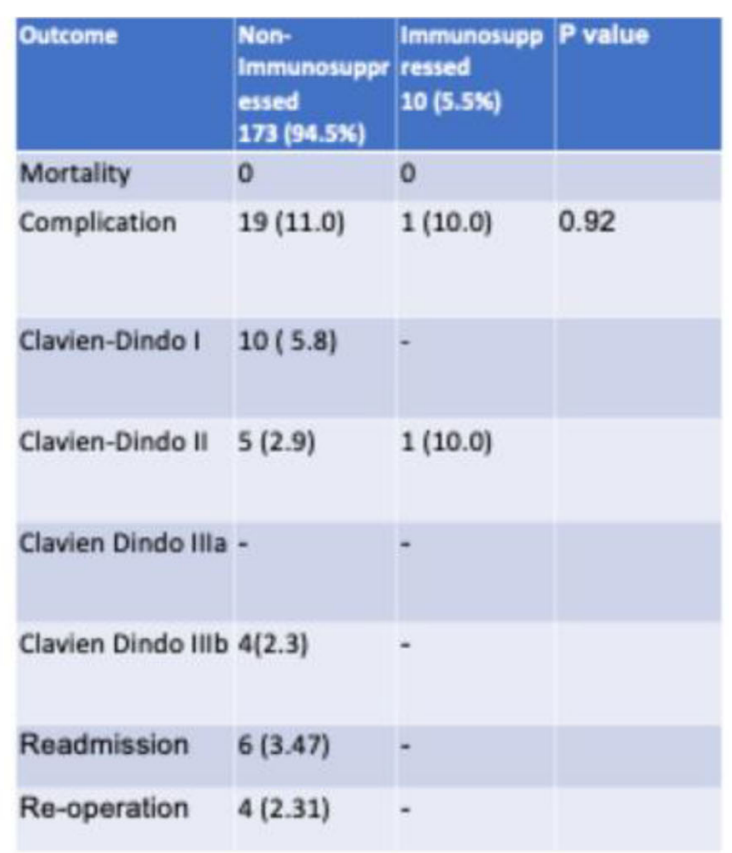

\section{Total Body Weight Loss \%}

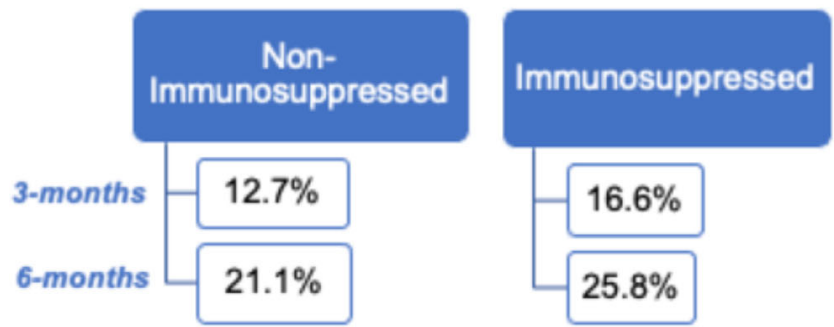

P19 - Remote tier 3 group programmes are an acceptable way to deliver preparatory dietetic and psychological intervention pre weight loss surgery and increase service capacity.

Miss Jenny Dacombe ${ }^{\mathbf{1}}$, Karen Coulman ${ }^{1}$, Rachel Elliott ${ }^{1}$, Marie Bird ${ }^{1}$, Jeanette Lamb ${ }^{1}$, Hañnah Mustard ${ }^{1}$, Aggie Mendieta-Tan ${ }^{1}$, Karen Buschenfeld, Jessica Munafo ${ }^{1}$, Lois Coy ${ }^{1}$

\section{${ }^{1}$ North Bristol Nhs Trust, Bristol, United Kingdom}

Background: Tier 3 group dietetic and psychological interventions are a key element of pre-bariatric surgery preparation. The COVID-19 pandemic has necessitated a shift to remote delivery. We evaluated the acceptability of a remote Tier 3 group intervention, and impact on service capacity.

Methods: Anonymous surveys were sent to 119 participants who completed an 8-week online tier 3 group programme (including pre-recorded 
and live sessions) between 17.08.20 and 15.04.21. The survey included 10 questions and focused on elements of the online group participants found helpful or unhelpful. Service capacity was assessed by maximum group session places offered.

Results: Thirty responses were received. $90 \%$ rated the group as helpful or very helpful and $93 \%$ would recommend to others. $83 \%$ had no technical difficulties; connectivity issues were the main complaint. $76 \%$ rated session duration satisfactory. Ongoing access to content and convenience were found helpful although informal interaction was missed. Maximum service capacity increased $40 \%$ by using pre-recorded content.

Conclusions: Remote Tier 3 group work was acceptable to most participants. Evaluation of patient outcomes is now needed. Remote group programmes will likely remain due to increased service capacity; reevaluation of acceptability post-pandemic and flexible modes of delivery may be beneficial.

P20 - Roux-en-y bypass patient information: An analysis of webbased information using the EQIP tool

Dr Saad Dalal ${ }^{\mathbf{1}}$, Mr Syme Bhopal ${ }^{2}$, Mr Naveed Hossain ${ }^{3}$, Mr Tanvir Hossain $^{1}$

${ }^{I}$ Nottingham University Hospitals NHS Trust, Nottingham, United Kingdom, ${ }^{2}$ University of Leeds, Leeds, United Kingdom, ${ }^{3}$ Whittington Hospital, London, United Kingdom

Background: Roux-en-y gastric bypass is a commonly performed procedure for weight loss with over 70,000 annually worldwide. Patient information quality is important for informed consent, expectations and compliance with post-operative plans. Awareness of the standard of online resources can guide development of information media.

Objective: To assess the quality of top-ranking patient information results on Google for Roux-En-Y gastric bypass.

Methods: The review was conducted using the search terms "Roux-en-y" into Google. The first 50 results were analysed using the Ensuring Quality Information for Patients (EQIP) tool.

Results: We calculated an EQIP score out of 100. Of 50 websites $40 \%$ had a score greater than $50,52 \%$ had a score between 25 and $49,8 \%$ had a score less than 25 which would represent a poor-quality resource. Median EQIP score was 45.5 (IQR 22.1). Mean was $46.9( \pm 11.9)$. Only $20 \%$ of websites discussed alternative treatments. Only $16 \%$ discussed how complications can be managed and $10 \%$ of sites quantified risks.

Conclusions: This study shows the top-ranking resources to be of a poor standard, particularly regarding the risk of complications and their management. Many failed to mention alternate treatment options. There is a need to provide higher quality resources for patient education.

P22 - Duodenal switch online patient information: an analysis using the EQIP tool

Dr Tal Ellenbogen ${ }^{1}$, Mr Tanvir Hossain ${ }^{1}$, Mr Naveed Hossain ${ }^{2}$

${ }^{1}$ Nottingham University Hospitals NHS Trust, Nottingham, United Kingdom, ${ }^{2}$ Whittington Health NHS Trust, London, United Kingdom

Background: Duodenal Switch is a drastic procedure for weight loss. When patients choose between this procedure and other interventions, reliable information is paramount for informed consent. It is therefore important to review the quality of this information available online due to the unregulated sphere that is the World Wide Web.

Objective: To assess the quality of publicly available online information on the duodenal switch operation.

Methods: We reviewed online material regarding duodenal switch using the search term "duodenal switch" in google. The top 100 results that met the inclusion/exclusion criteria were scored using the validated EQIP tool.
Results: 76 of the first 100 hits met the eligibility criteria. The overall median EQIP was 13.5; mean was 13.3. The scores ranged from 9 to 19 . $72.4 \%$ of websites were from the USA. Most websites were those of private bariatric clinics that provided patient information as well as marketing.

Conclusions: Based on the original EQIP tool used, the quality of the websites is extremely varied, with most being substandard. However, with a more focused version of the EQIP tool it would become clear that there is insufficient objective information pitched at the right level for patients.

P23 - Prevalence of stigmatising language in bariatric-metabolic surgery journals

Ms Naomi Fearon ${ }^{1}$, Mr Dimitri Pournaras ${ }^{2}$, Professor Carel le Roux ${ }^{3}, \mathrm{Mr}$ Richard Welbourn ${ }^{4}$

${ }^{1}$ Royal College of Surgeons, Dublin, Ireland, ${ }^{2}$ Southmead Hospital, Bristol, UK, ${ }^{3}$ University College Dublin, Dublin, Ireland, ${ }^{4}$ Musgrove Hospital, Taunton, UK

Background: People with obesity are subject to prejudice and stigma, perpetuated by use of potentially harmful terms. We aimed to summarise negative terminology reported in bariatric journals.

Methods: A systematic literature search of studies published in 2019 including terminology referring to words 'fail', 'morbid', and 'bariatric surgery'. Text from included articles was analysed. Papers were categorised into primary and revision articles and the use of 'fail' compared between groups. Descriptive statistics and odds ratio were performed to analyse association between type of papers and negative language. The use of the term 'morbid' was examined and prevalence was recorded.

Results: Out of a total of 3,020 screened papers, 71 (2.4\%) included the term 'fail'. Forty $(56 \%)$ referred to revision surgery. The odds ratio of 'fail' appearing in a study of revision surgery was 38.9 (95\% CI 22.1-70.2, $\mathrm{p}<0.0001$ ). Nineteen separate definitions of 'fail' were identified (table), with most $(15 / 19,79 \%)$ including an aspect of weight loss. The term 'morbid' used in conjunction with obesity was found in 508 papers $(16.8 \%)$.

Conclusions: This is the first study to examine the use of the terms 'fail' and 'morbid' in relation to bariatric surgery. There was a strong association between 'fail' and revision surgery.

Table 1. Definitions of 'fail' in the included studies a

EWL, excess weight loss; IWL, insufficient weight loss; WR, weight regain; TBW, total body weight; TWL, total weight loss; T2DM, type 2 diabetes mellitus; BMI, body mass index; VBG, vertical banded gastroplasty. a Note in some papers more than one definition was used

\section{Definition}

Times used

$\mathrm{EWL}<50 \%$

IWL or WR not defined 22

Adjustable gastric band-related complications 12

WR not defined

11

IWL not defined 8

'Weight loss failure' not defined 7

EWL $<50 \%$ and BMI $>35 \mathrm{~kg} / \mathrm{m}^{2}$, failure of endoscopic 1 each management of leak, failure of improvement in $\mathrm{HbAlc}$, intractable vomiting after VBG, IWL $<5 \%$ of TBW after 3 months or WR, less than $20 \%$ or $30 \%$ EWL, less than $5 \%$ or $10 \%$ TWL, minimal WL and intractable belching, no weight loss, recurrence of T2DM with WR, WR specified in case report 
P24 - Endoscopic ultrasound-directed trans-gastric ERCP (EDGE): Only a stone's throw away

Dr George Hiner ${ }^{1}$, Dr Panagiotis Vlavianos ${ }^{1}$, Dr Chris Wadsworth ${ }^{1}$, Dr Daniel Galloway ${ }^{7}$, Dr Salman Ahmed ${ }^{1}$, Mr Sanjay Purkayastha ${ }^{1}$, Dr Shwan Karim ${ }^{1}$, Dr Devinder Bansi ${ }^{1}$

\section{${ }^{1}$ Imperial College Healthcare NHS Trust, London, United Kingdom}

Obesity and weight loss are independent risk factors for cholelithiasis following bariatric surgery. Choledocholithiasis that require common bile duct clearance (CBD) can be complicated due to altered anatomy following Roux-en-Y gastric bypass preventing routine endoscopic retrograde cholangiopancreatography (ERCP). Alternate management options include laparoscopic-assisted ERCP, on table trans-cystic duct clearance and endoscopic ultrasound directed trans-gastric ERCP (EDGE). EDGE allows for safe CBD clearance through ERCP via an AXIOS stent placed with endoscopic ultrasound between the gastric pouch and the remnant stomach. This technique has a high clinical and technical success whilst being cost effective.

We present a case of a 58 year old lady who underwent Roux-enY gastric bypass for morbid obesity with associated type 2 diabetes mellitus and non-alcoholic fatty liver disease in 2012. She presented with abdominal pain in February 2021 and diagnosed with cholangitis secondary to choledocholithiasis. She underwent an EDGE procedure and following a successful gastric stent placement she returned 21 days later for an ERCP where the CBD was cleared of stones. After stent removal, the gastro-gastric fistula was closed using the Apollo OverStitch device to prevent her regaining weight. She was discharged with urgent surgical follow up for a cholecystectomy.

P27 - Patients' views and experiences of a live supervised tele-exercise programme following bariatric surgery during the COVID-19 pandemic: the BARI-LIFESTYLE qualitative study

Friedrich Jassil ${ }^{\mathbf{1}, \mathbf{2}, \mathbf{3}}$, Rebecca Richards ${ }^{4}$, Neville Lewis ${ }^{5}$, Alisia Carnemolla ${ }^{1,2,3}, \overline{\text { Gemma Montagut-Pino }}{ }^{1,2,3}$, Roxanna Zakeri ${ }^{1,2,3,7}$ Jessica Mok ${ }^{1,2,3,6}$, Kusuma Chaiyasoot ${ }^{1,2}$, Janine Makaronidis ${ }^{1,2,3}$, Kalpana Devalia ${ }^{6}$, Chetan Parmar $^{7}$, Rachel Batterham ${ }^{1,2,3}$

${ }^{1}$ Centre for Obesity Research, University College London, London, United Kingdom, ${ }^{2}$ Bariatric Centre for Weight Management and Metabolic Surgery, University College London Hospital NHS Trust, London, United Kingdom, ${ }^{3}$ National Institute for Health Research, UCLH Biomedical Research Centre, London, United Kingdom, ${ }^{4} M R C$ Epidemiology Unit, University of Cambridge, Cambridge, United Kingdom, ${ }^{5}$ The Hatter Cardiovascular Institute, Institute of Cardiovascular Science, University College London, London, United Kingdom, ${ }^{6}$ Bariatric Surgery Department, Homerton University Hospital NHS Trust, London, United Kingdom, ${ }^{7}$ Department of Surgery, Whittington Health NHS Trust, London, United Kingdom

Introduction: The BARI-LIFESTYLE trial is a randomised controlled trial evaluating the efficacy of a post-surgery nutritional-behavioural telecounselling and supervised exercise to maximise the health benefits of bariatric surgery. Due to the coronavirus disease 2019 (COVID-19) pandemic, the in-person exercise had to be converted to remote tele-exercise. However, patients' acceptability of this method of exercise provision is unknown.

Methods: Between 3-6 months following bariatric surgery, 13 adults participated in a weekly structured, 60-minute supervised exercise classes delivered via a video conferencing platform by a trained exercise therapist. 12 participants ( $n=8$ female; mean age $46 \pm 10$ years) who had undergone either sleeve gastrectomy $(n=8)$ or Roux-en-Y gastric bypass $(n=4)$ surgery participated in one-to-one semi-structured interviews following the programme. Interviews were audio-recorded, transcribed verbatim, and analysed using thematic analysis.

Results: Five key themes emerged: i) tele-exercise helped participants to cope with the impact of COVID-19 lockdown, ii) the acceptability of teleexercise, iii) the importance of professional support and advice, iv) selfregulation of exercise, and v) physical, emotional and social benefits of tele-exercise.

Conclusions: The tele-exercise was perceived as effective and useful as in-person exercise. These findings will inform future programmes that aim to integrate tele-exercise as part of the bariatric care pathway

\section{P28 - Revisional Bariatric Surgery: An Escalation in Surgical Management of Obesity}

Dr Emma C Kearns ${ }^{1}$, Ms Naomi M Fearon ${ }^{1}$, Dr Sadbh Brennan ${ }^{1}$, Dr Susannah Staunton ${ }^{1},{ }^{-}$r Kin Cheung $~ N g^{1}$, Professor Helen M Heneghan ${ }^{1}$

${ }^{1}$ St Vincent University Hospital, Dublin, Ireland

Background: The aim of this study was to assess perioperative and weight loss outcomes of revisional bariatric surgery (RBS) in an Irish bariatric patient cohort.

Methods: Data on consecutive revisional bariatric procedures was prospectively recorded and reviewed retrospectively. Data collected included demographic details, perioperative and weight loss outcomes.

Results: A total of 25 revisional bariatric procedures occurred in a 42-month period. Twenty-three patients underwent a second bariatric procedure, with two patients subsequently undergoing a third bariatric procedure. The primary bariatric procedures were gastric banding $(n=13,56.5 \%)$, sleeve gastrectomy $(\mathrm{N}=9,39.1 \%)$, gastric bypass $(\mathrm{N}=1,4.35 \%)$. The most common indications for RBS included weight regain $(68 \%, \mathrm{~N}=17)$, gastric band complications $(32 \%, \mathrm{~N}=8)$ and severe reflux symptoms $(20 \%, \mathrm{~N}=5)$. The majority of RBS patients were female $(\mathrm{N}=19,82.6 \%)$. Patient demographics are included in Table 1 . The revisional bariatric procedures were sleeve gastrectomy $(\mathrm{N}=5,20 \%)$, roux-en- $\mathrm{Y}$ gastric bypass $(\mathrm{N}=7,28 \%)$ and one-anastomosis gastric bypass $(\mathrm{N}=52 \%)$. There was no mortality recorded in this series. Overall postoperative morbidity rate was $16 \%(\mathrm{~N}=4)$. The 30 -day readmission and reoperation rates were $8 \%(\mathrm{~N}=2)$ and $4 \%(\mathrm{~N}=1)$, respectively. Total body weight lost (TBWL) at 12-months postoperatively was $17.25 \%$.

Conclusions: RBS is a safe and beneficial intervention for patients who regain weight or develop complications after primary bariatric surgery.

Table 1. Demographic Details

\begin{tabular}{ll}
\hline & $N=25$ \\
Age & $46.28 \pm 8.50$ years \\
& [Range 34-62] \\
& $82.6 \%$ Female \\
Gender & $126.86 \mathrm{~kg} \pm 31.05$ \\
Preop Weight & Range [71-190] \\
& $44.21 \mathrm{~kg} / \mathrm{m} 2 \pm 8.77 \mathrm{~kg}$ \\
Preop BMI & Range $[28.1-61]$ \\
& \\
COMPLICATIONS OF OBESITY & \\
Hypertension & $52 \%(\mathrm{~N}=12)$ \\
T2DM & $30.4 \%(\mathrm{~N}=7)$ \\
Dyslipidaemia & $39 \%(\mathrm{~N}=9)$ \\
Sleep Apnoea & $30.4 \%(\mathrm{~N}=7)$ \\
&
\end{tabular}


P29 - Internal hernia in short vs standard length biliopancreatic limb Roux-En-Y Gastric bypass: a review of a high-volume center of $\mathbf{1 0}$ years' experience

Dr Alwahhaj Khogeer ${ }^{2}$, Dr Faisal Alotaibi ${ }^{3}$, Dr Marco Adamo ${ }^{1}$, Dr Andrew Jenkinson ${ }^{1}$, Dr Mohamed Elkalaawy ${ }^{\mathrm{T}}$

${ }^{1}$ University College London Hospitals, London, United Kingdom, ${ }^{2}$ King Abdullah Medical City, Makkah, Saudi Arabia, ${ }^{3}$ King Fahad Medical city, Riyadh, Saudi Arabia

Background: In bariatric/metabolic surgery, Roux-en-Y gastric bypass (RYGB) remains one of the gold standard procedures. Post RYGB internal hernias affect around $4 \%$ of patients. It was hypothesized that having a shorter Biliopancreatic (BP) limb would decrease the risk of internal hernia. The aim of this analysis was to see how BP limb length affected the occurrence of internal hernia, regardless of mesenteric defects closure.

Objective: To review the effect of short compared to standard BP limb on incidence of post RYGB internal hernia.

Methods: A retrospective analysis of patients who underwent primary laparoscopic RYGB in a teaching university hospital was performed for the period between January 2010 and December 2020. Patients were divided into two groups: those with short BP limb $(40-50 \mathrm{~cm})$ without closure of mesenteric defects and those with standard BP limb (80-100 $\mathrm{cm}$ ) with mesenteric defects closure. The primary outcome was the development of an internal hernia.

Results: 691 cases of primary RYGB have been performed between 2010 and 2020. Of those, 429 patients had standard BP limb and 262 patients had short BP limb group. The overall incidence of internal hernia was $1.3 \%(n=9)$. There was no significant difference in the incidence between the two groups (6 patients in standard-limb group [1.39\%] vs 3 patients in short-limb group [1.14\%], $\mathrm{P}$ value $=0.53)$. Only one patient on each group required bowel resection due to ischemia (odds ratio $=0.611$ ). There was no internal hernia-related mortality in both groups.

Conclusion: In our experience, having a short BP limb did not seem to decrease the incidence of post RYGB internal hernia.

P30 - Laparoscopic Sleeve Gastrectomy: Shall we continue to perform histopathology examination of the resected stomach?

Miss Sylvia Krivan_ ${ }_{-}^{1}$, Mr James Chean Khun $\mathrm{Ng}^{2}$, Mr Brijesh Madhok ${ }^{1}$

${ }^{1}$ Royal Derby Hospital, Derby, United Kingdom, ${ }^{2}$ Queens Medical Centre, Nottingham, United Kingdom

Introduction: Laparoscopic Sleeve Gastrectomy(LSG) is a well-established bariatric procedure for morbid obesity. There has been ongoing debate regarding the value of routine histopathologic examination of LSG specimen.

Objectives: To examine our practice and discuss if a routine histology examination of the LSG specimen is necessary.

Methods: Retrospective analysis of all patients who underwent LSG at the Bariatric Unit at Royal Derby Hospital (09/2009-02/2020) was undertaken. Results: Some 358 patients underwent LSG in a consecutive period of 10.5years.Mean age was 46years.One hundred and nine(30.5\%)patients were male and $249(69.5 \%)$ female. For 84 patients $(23.5 \%)$ the specimen had not been sent for analysis. Amongst the remaining 274 patients,majority had no abnormality detected(N=181, 66.1\%).The most common histopathological changes were chronic gastritis in $61(22.3 \%)$ patients and benign gastric polyps in $22(8 \%)$, which did not require any further attention. Eight $(2.9 \%)$ patients were found to have Helicobacter pylori associated gastritis. More sinister findings were reported in $6(2.2 \%)$ patients, such as intestinal metaplasia( $(\mathrm{N}=2)$ and gastrointestinal stromal tumor (GIST)(N=4).All of these patients were older than 50 years and have been put on the surveillance list.
Conclusion: Our study shows that histopathologic examination of LSG specimen, especially in older patients, might harbor significant findings requiring further medical attention. This is relevant to inform patients and offer surveillance. We recommend histopathological examination of all LSG specimens and especially in patients over 50years of age.

\section{References:}

Tamer Safaan, Moataz Bashah, Walid El Ansari, and Mohsen Karam. Histopathological Changes in Laparoscopic Sleeve Gastrectomy Specimens: Prevalence, Risk Factors, and Value of Routine Histopathologic Examination. Obes Surg. 2017; 27(7): 1741-1749.

Maciej Walędziak, Anna Różańska-Walędziak, Michał R. Janik, Krzysztof W. Paśnik, and Piotr K. Kowalewski. Macroscopic Evaluation of Gastric Specimens After Laparoscopic Sleeve Gastrectomy - an Optimum Screening Test for Incidental Pathologies? Obes Surg. 2019; 29(1): 28-31.

Khaldoon Aljerian. Histopathological Findings in Laparoscopic Sleeve Gastrectomy Specimens from Patients with Obesity in Saudi Arabia. Gastroenterol Res Pract. 2018; 2018: 1702705.

P31 - Quality of life improvements post bariatric surgery in reproductive age women with and without PCOS.

Miss Hester Lacey ${ }^{1}$, Dr Stephanie Attersley-Smith ${ }^{2}$, Dr Sophia Stone ${ }^{2}$, Dr Kathryn Hart ${ }^{3}$, Dr Martin Whyte ${ }^{4}$, Professor Jill Shawe ${ }^{5}$

${ }^{1}$ Brighton and Sussex University Hospitals Trust, Brighton, United Kingdom, ${ }^{2}$ Western Sussex Hospitals NHS Foundation Trust, Chichester Medical Education Centre,, Chichester, United Kingdom. , ${ }^{3}$ Senior Lecturer in Nutrition and Dietetics, Faculty of Health and Medical Sciences, University of Surrey, , UK, ${ }^{4}$ Senior Lecturer in Metabolic Medicine, Department of Clinical \& Experimental Medicine, University of Surrey, , UK, ${ }^{5}$ Director of IHC and Professor in Women's Health, Faculty of Health, School of Nursing and Midwifery, University of Plymouth/ Honorary Professor at University of Surrey, , UK

Obesity is a disease that has severe consequences on physical and psychological health and quality of life (QOL). Polycystic Ovarian Syndrome (PCOS), closely associated with obesity, presents with a syndrome of subfertility, hyperandrogenism, and abnormal metabolism. This study looks at QOL before and after bariatric surgery in women with and without PCOS, to determine the impact of PCOS on QOL, and how weight loss can improve QOL.

Participants completed questionnaires exploring health-related quality of life (HR-QoL) prior to surgery and at three, six and twelve months after surgery. Weight loss and improvement of clinical symptoms was assessed and compared to QOL improvements to identify factors impacting QOL. For both groups, bariatric surgery resulted in significant QOL improvements. Symptoms of irregular/absent periods and hirsutism, showed significant improvements in both groups, and were related to QOL improvements.

The beneficial effects of bariatric surgery on QOL indicate that early consideration of surgery in these patients will likely provide significant and sustained clinical benefit. Resolution of clinical symptoms of PCOS with weight loss indicate how weight loss may ameliorate the syndrome of PCOS. The study results improve understanding of the impacts of PCOS on QOL and can be used to improve their clinical management.

P32 - Roux-en-Y gastric bypass and Sleeve Gastrectomy exhibit energy expenditure equally one-year post-surgery

Dr Khalefah Malallah,2, Dr Kleopatra Alexiadou ${ }^{1}$, Ms Yasmin Tabbakh ${ }^{1}$, Mr Ahmed Ahmed ${ }^{1}$, Mr Sanjay Purkayastha ${ }^{1}$, Mr Christos Tsironis ${ }^{1}$, Mr Sherif Hakky ${ }^{1}$, Dr Harvinder Chahal ${ }^{1}$, Professor Tricia Tan ${ }^{1}$ 
${ }^{1}$ Imperial College London, , United Kingdom, ${ }^{2}$ Jaber Al-Ahmed Armed Forces Hospital of Kuwait, Kuwait City, Kuwait

Background: Changes in various components of energy expenditure (EE), such as resting energy expenditure (REE) and diet-induced thermogenesis (DIT) may influence the long-term maintenance of weight loss after bariatric surgery. Roux-en-Y gastric bypass (RYGB) and Sleeve Gastrectomy (SG) are two widely used procedures with similar efficacy in inducing sustainable weight loss.

Aim: To compare the REE, DIT and REE: FFM (Fat Free Mass) ratio between patients undergoing RYGB or SG at more than one-year post surgery.

Methods: Sixteen patients ( 8 RYGB and 8 SG) were invited for a mixed meal test (MMT) at more than one-year post-surgery. EE was measured before and after 1 hour of the MMT ingestion using calorimetry. Body composition was measured using Tanita BC-418MA body composition analyser. Unpaired t test was used to compare the two groups.

Results: There was no significant difference between the two groups in terms of weight, HbA1c, REE, DIT and REE: FFM ratio $(p=0.222$, $\mathrm{p}=0.525, \mathrm{p}=0.678 \mathrm{p}=0.759$ and $\mathrm{p}=0.098$ respectively). On the other hand, there was a significant increase in DIT in each of the RYGB and SG group ( $\mathrm{p}=0.0002$ and $\mathrm{p}=0.0018$ respectively).

Conclusion: Our preliminary findings demonstrated that there is no difference in neither REE nor DIT between RYGB and SG at more than oneyear post-surgery suggesting similar effect of both procedures on EE. Further studies with a larger sample size and longer term follow up are required to investigate whether a difference in the various components of EE between the two surgeries could play a role in weight loss maintenance and prevention of weight regain.

P34 - An audit of the psychological factors related to feeding complications post bariatric surgery.

\author{
Miss Alice Pace_, Dr Anna Smith ${ }^{1}$ \\ ${ }^{1}$ Chelsea And Westminster Hospital, London, United Kingdom
}

An audit investigating whether individuals who experience PPS (persistent physical symptoms) post bariatric surgery, and such require a feeding tube, received the standard of psychological care outlined by the BPS (2019). PPS literature indicates that trauma, childhood abuse and illness specific stressors may be important factors in the development of PPS symptoms.

Participants identified from bariatric database, $n=5$ met inclusion criteria with a control group $(n=5)$ of participants randomly selected from the same database. Participant's notes on NHS clinical management systems were analysed using descriptive statistics outlining the psychological input received. Notes further reviewed to ascertain history of trauma, childhood abuse and illness specific stressors.

Across all groups, $0 \%$ of participants met the standard of psychological care outlined by the BPS. Further exploration showed that $80 \%$ of the PPS group experienced trauma, $20 \%$ child abuse and $100 \%$ illness specific stressor. Control group results showed 20\% trauma, 20\% child abuse, $20 \%$ illness specific stressor.

None of the patients audited met the predetermined standard of psychological care (BPS, 2019). This exploratory study indicates that having an illness specific stressor is correlated with post-surgical feeding complications. This audit highlights the need to enhance the psychological support pre bariatric surgery so to potentially mitigate post-surgery complications.

$\begin{array}{ll}\text { Psychological } & \text { Psychological } \\ \text { intervention lasting } & \text { intervention guided by } \\ \text { a minimum of } 6 & \text { shared integrative } \\ \text { months } & \text { formulation }\end{array}$

(continued)

\begin{tabular}{lll}
\hline Patients requiring & $0(0 \%)$ & $0(0 \%)$ \\
feeding tube post & \\
bariatric surgery & \\
$(\mathrm{n}=5)$ & \\
\hline
\end{tabular}

\begin{tabular}{llll}
\hline & Trauma & Childhood abuse & Illness specific stressor \\
$\begin{array}{l}\text { Patients requiring } \\
\text { feeding tube }\end{array}$ & $4(80 \%)$ & $1(20 \%)$ & $5(100 \%)$ \\
$\begin{array}{c}\text { post bariatric } \\
\text { surgery }(\mathrm{n}=5)\end{array}$ & & & \\
\hline
\end{tabular}

\begin{tabular}{|c|c|c|c|}
\hline \multicolumn{2}{|c|}{$\begin{array}{l}\text { Patients who have not } \\
\text { required a feeding tube } \\
\text { post bariatric surgery } \\
(\mathrm{n}=5)\end{array}$} & $\begin{array}{l}\text { Psychological } \\
\text { intervention } \\
\text { lasting a minimum } \\
\text { of } 6 \text { months }\end{array}$ & $\begin{array}{l}\text { Psychological } \\
\text { intervention } \\
\text { guided by a shared } \\
\text { formulation }\end{array}$ \\
\hline & Trauma & $\begin{array}{l}\text { Childhood } \\
\text { abuse }\end{array}$ & $\begin{array}{l}\text { Illness } \\
\text { specific } \\
\text { stressor }\end{array}$ \\
\hline $\begin{array}{l}\text { Patients who } \\
\text { have not } \\
\text { required a } \\
\text { feeding tube } \\
\text { post bariatric } \\
\text { surgery } \\
(\mathrm{n}=5)\end{array}$ & $1(20 \%)$ & $1(20 \%)$ & $1(20 \%)$ \\
\hline
\end{tabular}

\section{References}

The British Psychological Society. Psychological perspectives on obesity: Addressing policy, practice and research priorities. 2019.

P35 - Surgical Intervention for Gastro-Oesophageal Reflux after One Anastomosis Gastric Bypass - A Systematic Review

Mr Nayer Rizkallah ${ }^{1}$, Ms Rachel Lee ${ }^{1}$, Mr Brijesh Madhok ${ }^{1}$

${ }^{1}$ University Hospitaīs of Derby and Burton, , United Kingdom

Introduction: One Anastomosis Gastric Bypass (OAGB) is now a mainstream bariatric procedure. However, intractable gastro-oesophageal reflux disease (GORD) after OAGB might necessitate revisional surgery. We reviewed the current literature on revisional surgery after OAGB for GORD.

Methods: This systematic review followed the Preferred Reporting Items for Systematic Reviews and Meta-analyses (PRISMA) guidelines via a multi-database (MEDLINE, EMBASE and PubMed) systematic search. Results: Out of 989 studies identified, 17 were included; 1 randomised controlled trial, 14 case series and 2 case reports. Out of 10,133 OAGB patients, $105(1 \%)$ patients underwent revisional surgery for GORD. Clinical presentations and investigations were scarcely reported. In four studies (41 patients), revisional surgery was performed after a mean of 28.2 months from OAGB. Revision 
to Roux-En-Y configuration was done in $99(94.2 \%)$ patients; $42(42.4 \%)$ had Lonroth technique, 12 (12.1\%) had gastrojejunostomy resected while in $45(45.5 \%)$ the technique wasn't mentioned. Six $(5.8 \%)$ patients had Braun entero-enterostomy added to their OAGB. Reflux resolved in $45(42.8 \%$ ) patients, persisted in $2(1.9 \%)$ patients and was not reported in $58(55.3 \%)$ patients. Conclusion: Although firm conclusions are difficult given the gaps in the current literature, reported revisional surgery after OAGB for GORD is rare. Conversion to Roux-en-Y configuration is the commonest procedure.

P36 - Does bariatric surgery result in improvement of liver function tests in people affected by obesity?

Dr James Sandell_, Mr Zaher Toumi ${ }^{1}$

${ }^{1}$ County Durham And Darlington Nhs Foundation Trust, , United Kingdom

Background: NAFLD is a common condition among people with obesity. The only effective treatment is weight loss. This study aims to find out if bariatric surgery can improve some of the biochemical parameters of NAFLD.

Methods: A retrospective analysis of prospective database of patients who had bariatric surgery over 4-year period. The most recent liver function tests were compared to the last set of tests done preoperatively.

Results: 108 consecutive patients underwent bariatric surgery by a single surgeon over a 4-year period. 102 were included in the study (51 sleeve gastrectomy and 51 Roux-en-Y gastric bypass). 78 were female and 24 were male, with a median age of 46 . There was a significant decrease in median ALT post surgery $(26 \mathrm{u} / \mathrm{l}$ to $23 \mathrm{u} / \mathrm{l}, \mathrm{p}=0.02)$. When analysing patients who had LSG and RYGB separately, there was no significant difference in ALT levels in the RYGB cohort while there was a significant decrease in ALT in the LSG cohort. There was not a significant change in ALP and Bilirubin levels in the whole cohort and in the subgroups.

Conclusions: Bariatric surgery results in improvement of some of the biochemical markers of NAFLD. The effect is more pronounced in patients who undergo sleeve gastrectomy.

P38 - Long-term follow-up after sleeve gastrectomy versus Roux-en$Y$ gastric bypass versus mini gastric bypass: a prospective randomized comparative study of weight loss and remission of comorbidities:

Ms Deeba Siddiqui ${ }^{1}$, Dr. Arun Prasad ${ }^{1}$

${ }^{1}$ Apollo Hospital, New Delhi, India

Long-term follow-up after sleeve gastrectomy versus Roux-en-Y gastric bypass versus mini gastric bypass: a prospective non-randomized comparative study of weight loss and remission of comorbidities

Background: Bariatric Surgery has exponentially increased in the last decade, as it is associated with very low complications, mortality, readmissions and reoperations rates, and shows excellent short- and long-term benefits of weight loss and resolution of comorbidities. The aim of this study was to compare the effect of SG, RYGB, and OAGB, on short- and long-term weight loss and comorbidities resolution.

Methods: A prospective non randomized clinical study of all morbidly obese patients undergoing SG, RYGB, and MGB, as primary bariatric procedures, was performed. Patients were randomly assigned into 3 groups as per their choice after explaning all procedures. BMI, excess BMI loss (EBMIL) and remission of type 2 diabetes (T2DM), hypertension (HT), and dyslipidemia (DL) were assessed.

Results: 150 patients were included in the study, 50 in each group. First 50 of each procedure were taken after the start date.Follow-up rate at 3 years postoperatively was $91 \%$ in SG group, $92 \%$ in RYGB, and $90 \%$ in
MGB. MGB achieves significantly greater EBMIL than RYGB and SG at 1,2 , and 3 years $(\mathrm{p}<0.001$, respectively). At years, MGB achieves significantly greater remission of T2DM $(p=0.027)$, HT $(p=0.006)$, and DL ( $p<0.001)$ than RYGB and SG. RYGB did not show significant superiority than SG in short- and long-term remission of T2DM and HT, but achieves greater remission of DL $(p<0.001)$.

Conclusion: MGB achieves superior mid- and long-term weight loss than RYGB and SG. There are no significant differences in weight loss between SG and RYGB at 1, 2, and 3 years. MGB achieves better short- and long-term resolution rates of DM, HT, and DL than SG and RYGB. RYGB reaches significantly greater rates of T2DM \& DL remission than SG.

P39 - Gastric bypass is an effective treatment for individuals with Dercum's disease and obesity: A case report

Ms Jeanette Lamb_, Mr Naim Slim ${ }^{1}$, Mr James Hewes ${ }^{1}$, Mr Dimitri Pournaras $^{1}$

\section{${ }^{1}$ North Bristol NHS Trust, Bristol, United Kingdom}

Introduction: Dercum's disease is a rare condition characterised by the extensive presence of multiple painful lipomas, with which there is a strong association with morbid obesity. Weight loss in affected patients is refractory to lifestyle modifications, and evidence for the efficacy of weight loss surgery is limited. We report successful weight loss after Roux-en-Y gastric bypass (RYGB) in a patient with Dercum's disease.

Case Report: A 48 year old lady presented for consideration for weight loss surgery. Her co-morbidites included irritable bowel syndrome, eczema, gastrooesophageal reflux disease, and Dercum's disease for which she has had several lipoma excisions. Her body mass index (BMI) at presentation was $48 \mathrm{~kg} / \mathrm{m}^{2}$. Prior to her surgery, she underwent full multidisciplinary evaluation. She underwent laparoscopic RYGB in November 2019 , using a standard five-port approach, a $20 \mathrm{~cm}^{3}$ gastric pouch, $80 \mathrm{~cm}$ biliopancreatic limb, and $120 \mathrm{~cm}$ alimentary limb. There were no complications, and she was discharged on the following day. At her sixteenmonth follow-up, her BMI reduced dramatically to $34 \mathrm{~kg} / \mathrm{m}^{2}$.

DiscussionThe role of surgery in patients with Dercum's disease is controversial, with one published case report reporting obesity resistant to RYGB with no weight loss effect postoperatively. This report suggests that people with Dercum's can respond to RYGB.

P42 - A quantitative investigation into the impact of bariatric surgery on HbA1c levels, diabetes-related medication and weight in obese patients with type 2 diabetes and preoperative BMI of $>35 \mathrm{~kg} / \mathrm{m} 2$.

\section{Miss Hannah Van Tromp_, Dr Xenofon Tzounis ${ }^{1}$ \\ ${ }^{1}$ University of Hertfordshire, School of Life and Medical Sciences, , United Kingdom}

Background: Despite the prevalence of obesity and type 2 diabetes (T2DM) continuing to rise globally, rates of bariatric surgery remain low. Further research is needed to present the impact of bariatric surgery on the management of T2DM in people with obesity and T2DM.

Methods: Patient demographics and clinical data were collected retrospectively from 52 adults with obesity and T2DM who underwent bariatric surgery in 2018. Statistical tests were conducted to compare pre- and postoperative data and to investigate associations, $p=<0.05$ deduced statistical significance.

Results: The majority of the cohort were female (76.9\%) and had had gastric bypass procedure $(81.5 \%)$. Mean \pm SD age was $53.6 \pm 10.6$ years and duration of diabetes mellitus was $6.9 \pm 7.6$ years. Mean preoperative weight dropped from $125 \pm 22 \mathrm{~kg}$ to $96 \pm 17 \mathrm{~kg}$ postoperatively and $\mathrm{HbAlc}$ from $55.2 \pm 9.5 \mathrm{mmol} / \mathrm{mol}$ to $42 \pm 10 \mathrm{mmol} / \mathrm{mol},(\mathrm{p}=<0.0005) .76 .9 \%$ of participants were on diabetes-related medication preoperatively, this 
dropped to $26.9 \%$ postoperatively. Moderate negative correlations were found between duration of diabetes mellitus and postoperative medication reduction $(\mathrm{rs}(98)=-0.327, \mathrm{p}=<0.039)$ and $\mathrm{HbA} 1 \mathrm{c}(\mathrm{mmol} / \mathrm{mol})$ reduction $(\mathrm{rs}(98)=-0.239)$.

Conclusion: Our findings suggest that early bariatric surgical intervention could lead to improved management of T2DM in people with obesity and T2DM.

P45 - Once-weekly subcutaneous semaglutide $2.4 \mathrm{mg}$ reduces body weight in adults with overweight or obesity regardless of baseline characteristics (STEP 1)

W Timothy Garvey ${ }^{2}$, Dan Hesse ${ }^{3}$, Professor Frank Joseph, Anna Koroleva $^{3}$, Robert F Kushner ${ }^{1}$, Carel W le Roux ${ }^{8}$, Soo Lim ${ }^{4}$, Ildiko Lingvay $^{5}$, Ofri Mosenzon ${ }^{6}$, Signe OR Wallenstein ${ }^{3}$, Thomas A Wadden ${ }^{7}$

${ }^{1}$ Division of Endocrinology, Feinberg School of Medicine, Northwestern University, , USA, ${ }^{2}$ Department of Nutrition Sciences, University of Alabama at Birmingham, Birmingham, , USA, ${ }^{3}$ Novo Nordisk A/S, Søborg, , , Denmark, ${ }^{4}$ Department of Internal Medicine, Seoul National University College of Medicine, Seoul National University Bundang Hospital, Seongnam, , Korea, Republic of, ${ }^{5}$ UT Southwestern Medical Center, Dallas, , United States, ${ }^{6}$ Diabetes Unit, Department of Endocrinology and Metabolism, Hadassah Medical Center, Faculty of Medicine, Hebrew University of Jerusalem, Ein Kerem, , Israel, ${ }^{7}$ Department of Psychiatry, Perelman School of Medicine, University of Pennsylvania, , Philadelphia, USA, ${ }^{8}$ Diabetes Complications Research Centre, Conway Institute, University College Dublin, Dublin, Ireland

Introduction: This post hoc analysis of STEP 1 investigated weight loss according to baseline characteristics.

Methods: In STEP 1, adults with overweight or obesity were randomised 2:1 to 68 weeks' treatment of once-weekly subcutaneous semaglutide $2.4 \mathrm{mg}$ or placebo (+lifestyle intervention). Adults (aged $\geq 18$ years) had BMI $\geq 27 \mathrm{~kg} / \mathrm{m} 2$ and $\geq 1$ weight-related comorbidity or BMI $\geq 30 \mathrm{~kg} / \mathrm{m} 2$, without T2D. Categorical weight loss (baseline-week 68) was evaluated descriptively by baseline characteristics (age, sex, race, body weight [BW], BMI, waist circumference, glycaemic status). Mean percent weight loss (baseline-week 68) was analysed by sex and baseline BW using MMRM analysis.

Results: STEP 1 included 1961 participants (BW 105.3kg, BMI 37.9kg) $\mathrm{m} 2$ ). At Week 68, proportions of participants with $\geq 20 \%, 15-<20 \%, 10$ $<15 \%$ and $5-<10 \%$ weight loss were $34.8 \%, 19.9 \%, 20.0 \%$ and $17.5 \%$ with semaglutide vs $2.0 \%, 3.0 \%, 6.8 \%$ and $21.2 \%$ with placebo, respectively. Participant distribution across weight loss groups appeared unaffected by baseline characteristics, except sex and baseline BW. Mean percent weight loss at week 68 with semaglutide was greater among females than males, and in participants with lower vs higher baseline BW. Conclusions: Weight loss with semaglutide occurred in all subgroups evaluated. Female sex and low baseline BW were associated with greater response to semaglutide.

Funding: Supported by Novo Nordisk

P46 - Revision of failed Vertical Banded Gastroplasty (VBG) to Rouxen-Y Gastric Bypass (RYGB)

\author{
Mr Ahmed Ghanem_, Mr Ahmed Ahmed ${ }^{1}$ \\ ${ }^{1}$ Imperial College Healthcare NHS Trust, , United Kingdom \\ Revision of failed Vertical Banded Gastroplasty (VBG) to Roux-en-Y \\ Gastric Bypass (RYGB)
}

Background: A lady in her fifth decade presented with ongoing weight regain after a vertical banded gastroplasty (VBG) operation 10 years ago. Due to the unsatisfactory results, the patient underwent Single Anastomosis Sleeve Ileal Bypass (SASI).
Methods: Careful dissection of the stapled part of the stomach and excision of the previous sialistic ring was done followed by the construction of a laparoscopic Roux-en-Y Gastric Bypass with a $50 \mathrm{~cm}$ BP limb and a 150 Roux limb in ante-colic ante-gastric fashion.

Results: The revision using laparoscopic RYGB was successful. There were no postoperative surgical complications and the patient progressed well postoperatively.

Conclusion: VBG used to be one of the popular options for weight loss surgery, but now it poses a challenge to be revised to a different operation due to adhesions and the risk of a leak. Laparoscopic RYGB can be used as the gold standard for the management of such cases to improve and maintain weight loss.

P47 - Why Gastric Bypass should not be done on Smokers: lifethreatening Bleeding marginal ulcer eroding to the remnant stomach

Dr Alwahhaj Khogeer ${ }_{-}^{2}$, Dr Mohamed Elkalaawy ${ }^{1}$, Dr Marco Adamo ${ }^{1}$, Dr Faisal Alotaibi ${ }^{1}$

${ }^{1}$ University College London Hospitals, London, United Kingdom, ${ }^{2}$ King Abdullah Medical City, Makkah, Saudi Arabia

Background: Laparoscopic Roux-En-Y gastric bypass LRYGBP is one of the most commonly performed bariatric procedures. Smoking increases the risk of thromboembolic events and marginal ulcers formation after gastric bypass. Smoking with marginal ulcers could lead to serious bleeding or perforation, both could be life-threatening. Smokers tend to take the risk lightly despite advice. Should gastric bypass be offered to a smoker? Method: In this video, we present a case of post gastric bypass lifethreatening upper GI bleeding from a marginal ulcer eroding to remnant stomach and tributaries of left gastric artery caused by smoking, and its acute management.

Results: 69 years old female. heavy smoker. Had LRYGBP in another center 9 years prior to her presentation. She was diagnosed with marginal ulcer on endoscopy 4 months prior to her presentation to us and she was put on medical treatment. She was advised to stop smoking; however, she did not comply.

She Presented to our hospital with melena and hematemesis with dropped Hemoglobin. She was admitted through A\&E and resuscitated. Urgent endoscopy was performed showing bleeding marginal ulcer with large clots and fresh blood. Could not definitely control bleeding. Patient collapsed in the ward, revived after extensive resuscitation. Patient was shifted for emergency surgery.

Procedure was performed laparoscopically. The bleeding ulcer was found eroding to the remnant stomach, the lesser omentum and to tributaries of left gastric artery. The ulcer was over-sewn, hemostasis was secured and the gastrojejunostomy was resected. A neo-gastrojejunostomy was created. Patient recovered well from the procedure with no complications or rebleed.

Conclusion: Acute management of bleeding marginal ulcers could be challenging. Smoking after gastric bypass could lead to life-threatening complications. Gastric bypass should not be offered to active smokers.

P48 - Duodenal switch revision: importance of intraoperative endoscopy

Miss Patricia M Ortega ${ }^{1}$, Mr Ahmed Ahmed ${ }^{1}$, Dr Devinder Bansi ${ }^{1}$, Dr Jonathan Cousins ${ }^{1}$

${ }^{1}$ Imperial Weight Centre, London, United Kingdom

Introduction: Several publications have recommended the routine use of intraoperative endoscopy to decrease complications in primary bariatric surgery. 
Given revisional surgery is basically more challenging, the use of intraoperative endoscopy should always be taken into account in order to prevent complications and/or improve outcomes.

Methods: - A 52 yo female with multiple Bariatric procedures since 2001 complained about severe reflux and weight regain. In fact, the last procedure was a duodenal switch in 2013.

- Barium meal showed images compatible with stenosis in her duodenoileal anastomosis whereas endoscopy demonstrated that the anastomosis was patent

- A diagnostic laparoscopy was performed to delineate the anatomy and try to fix both issues reflux and weight regain.

Results

- Distalization of the ileo-ileal anastomosis was performed in order to reduce common channel from $150 \mathrm{~cm}$ to $100 \mathrm{~cm}$.

- Intraoperative endoscopy : An angulation of the sleeve correlated with the laparoscopic view allowed us to focused on the problematic area and proceed to extensive adhesiolysis.

- Once the sleeve was totally free and mobile a new gastroscopy was performed and stomach looked straighter.

Conclusion: Intraoperative endoscopy allowed us to correlate anatomy in order to repair a mechanical problem.

P49 - Managing severe abdominal pain 3 years after RYGB: resection of marginal ulcer and primary GJ reconstruction

Miss Patricia M Ortega ${ }^{\mathbf{1}}$, Mr Ahmed Ahmed ${ }^{1}$, Mr Jose Maria Valencia ${ }^{2}$

${ }^{1}$ Imperial Weight Centre, , United Kingdom, ${ }^{2}$ Crownwell Hospital, London, United Kingdom

Introduction: The increasing incidence of obesity across the globe has led to a significant rise in the number of bariatric procedures per- formed for weight loss. Given laparoscopic Roux-en-Y gastric bypass is one of the commonest procedures performed worldwide, there is an increasing awareness of the complications associated with it including leak, hemorrhage, bowel obstruction, anastomotic stricture, gastro-gastric (GG) fistula formation, internal hernia and marginal ulcer.

Methods

A 40 year old female with a surgical history of RYGB 3 years ago presented as an emergency due to excruciating 5-day epigastric abdominal pain. As a consequence of the severity of her symptoms, she was directly taken to theatre to perform a diagnostic laparoscopy.

Results: After ruling out internal hernia, an intraoperative endoscopy was performed. Findings showed a big marginal ulcer so decision was made to excise it and re-do a primary reconstruction of the GJ anastomosis

Patient was discharged the day after: able to eat and drink and not complaining of any pain.

Conclusion: Although some post RYGB complications can be treated conservatively, it is necessary to tailor each patient's presenting complaint in order to make a good decision at the right moment.

\section{P50 - Revision Sleeve Gastrectomy after Failed Gastric Plication}

Miss Hiba Shanti_, Miss Amber Shivarajan ${ }^{1}$, Professor Ameet G. Patel ${ }^{1}$

${ }^{1}$ King's College Hospital, London, United Kingdom

Laparoscopic Gastric Plication (LGP) has been proposed as a less invasive and less costly alternative to sleeve gastrectomy. We present a 30-year-old lady with an initial weight of $160 \mathrm{Kg}$ (BMI $55 \mathrm{Kg} / \mathrm{m} 2$ ) who underwent LGP five years previously in Prague. She lost $50 \mathrm{Kg}$ in the first year, her lowest weight was $110 \mathrm{Kg}$. Unfortunately, her weight has gradually increased and is currently $148 \mathrm{Kg}$ (BMI $51 \mathrm{Kg} / \mathrm{m} 2)$. She is seeking further weight loss surgery. We believe to achieve her target weight of $70 \mathrm{Kg}$; she will require a malabsorptive procedure. Our approach was for a sleeve gastrectomy followed by a second stage SADI or DS if required.

Intra-operatively, the gastric wall plication was noted to be disrupted in the middle with herniation of the stomach between plication sutures. The sutures were incorporated by scarring and fibrosis, these were divided. The gastric plication was completely dismantled in preparation for safe stapling. The patient recovered well with no complications. She lost $15 \mathrm{Kg}$ in 6 weeks.

O1 - Feasibility of a multi-parametric continuous monitoring wearable device in patients undergoing bariatric surgery

Mr Kishan Patel ${ }^{1}$, Mr Ravi Aggarwal ${ }^{\mathbf{1 , 2}}$, Mr Guy Martin ${ }^{1,2}$, Mr Colin Bicknell $^{1,2}$, Mr Ahmed Ahmed ${ }^{1,2}$

${ }^{1}$ Imperial College London, London, United Kingdom, ${ }^{2}$ Imperial College Healthcare NHS Trust, London, United Kingdom

Introduction: Continuous, remote vital sign monitoring in the perioperative period may lead to earlier detection of patient deterioration. This study aimed to explore acceptability and accuracy of a wearable sensor for patients undergoing bariatric surgery.

Methods: A pilot prospective cohort study was undertaken at our bariatric unit in London. The sensor continuously measured heart rate (HR), temperature, respiratory rate (RR) and oxygen saturation. Accuracy was measured by comparing sensor readings with nurse readings while participants were inpatients, and acceptability was measured by daily usage and a patient satisfaction questionnaire.

Results: 12706 sensor and 221 nurse readings were obtained from eight patients. Mean length of use was $7.0 \pm 1.5$ days at an average of 104.7 hours per patient. High satisfaction scores were recorded from participants. The agreement was high in HR (mean bias $-0.3 \mathrm{bpm}, 95 \%$ limits of agreement (LOA) $-7.5 \mathrm{bpm}-6.9 \mathrm{bpm}$ ), moderate in RR (median bias 0.0 breaths $/ \mathrm{min}, 95 \% \mathrm{LOA}-5.5-13.2 \mathrm{breaths} / \mathrm{min}$ ), poor in temperature (mean bias $-1.9^{\circ} \mathrm{C}, 95 \% \mathrm{LOA}-5.5^{\circ} \mathrm{C}-3.3^{\circ} \mathrm{C}$ ) and poor in oxygen saturation (median bias $-2.0 \%, 95 \%$ LOA $-19.8 \%-3.5 \%$ ).

Conclusions: The device was well-received by all patients and reliably measured HR, but not any other observation. Despite the potential of remote monitoring devices, acceptability and accuracy must be further assessed in bariatric populations before widespread implementation.

O2 - Evaluation of Patient Centred Digital Information in Bariatric Surgery - a Content Analysis of Mobile Applications and Web-based Information.

Dr Syed Daniyal Ahsan_${ }_{-}^{1}$, Dr Nyima Sanyang ${ }^{1}$, Miss Alice Yi-Chien Tsai $^{1}$, Mr Sasindran Ramar ${ }^{1}$

${ }^{1}$ King's College Hospital, , United Kingdom

Introduction: Digital information serves as reference tools and intervention adjuncts in the care of Bariatric patients. This is invaluable during the Covid-19 Pandemic, however carries a wide range of content variations. The study aims to evaluate bariatric websites and Mobile Apps relevant to UK population.

Methods: Thematic analysis was applied to British Obesity \& Metabolic Surgery Society (BOMSS), National Institute for Health and Care Excellence (NICE), American Society for Metabolic and Bariatric Surgery (ASMBS) websites and a Bariatric Mobile App with 2 independent reviewers. The variation saturation was reached and used as comparators to additional 6 Mobile Apps identified in a systematic approach.

Results: Fourty-seven comparators ( 22 pre-operative, 2 peri-operative, 5 post-operative, 8 patient journey and 10 others) were applied to 10 
resources. BOMSS (41\%) and ASMBS (62\%) contained the most information, however, don't incorporate patient journey. $14.3 \%$ of Mobile App provides monitoring and journey tracking in pre-operative and postoperative care; $29 \%$ were funded and advertised by private clinics.

Conclusion: There is a wide variation of digital content currently available to bariatric patients.

There are 2 websites and no Mobile App that are specific to UK patients. This study highlight the needs for standardisation and development an interactive digital platform.

\section{O4 - Can current methods of predicting T2D remission following metabolic surgery be improved?}

Miss Iona Davies ${ }^{1}$, Dr Julia Kenkre ${ }^{1}$, Dr Kleopatra Alexiadou ${ }^{1}$, Dr Khalefah Malallah ${ }^{\top}$, Dr Yemi Ilesanmi ${ }^{1}$, Dr Saleem Ansari ${ }^{1}$, Dr Anna Kamocka $^{1}$, Dr Harvey Chahal ${ }^{2}$, Mr Christos Tsironis ${ }^{2}$, Mr Sherif Hakky $^{2}$, Mr Ahmed Ahmed ${ }^{2}$, Mr Sanjay Purkayastha ${ }^{2}$, Professor Tricia $\operatorname{Tan}^{1}$

${ }^{1}$ Section of Investigative Medicine, Imperial College London, London, United Kingdom, ${ }^{2}$ Department of Surgery, Imperial College Healthcare NHS Trust, London, United Kingdom

Background: DiaREM and ABCD are validated scores for predicting T2D remission following bariatric surgery. However, these scores were generated in ethnically homogeneous populations and are poorly predictive in an ethnically diverse population.

Aim: To identify additional parameters that improve the performance of pre-existing remission scores in an ethnically diverse population from the UK.

Method: Prospective analysis of 83 patients with T2D who underwent Roux-en-Y gastric bypass (RYGB). Remission (complete and partial) was judged using ADA criteria at 1-year after surgery. The performance of DiaREM was compared against ABCD using Area-under-curve for receiver-operator-characteristic (AUCROC). This was followed by modelling with the inclusion of other demographic parameters and biomarkers derived from a baseline mixed meal test (MMT). Prediction improvement was assessed via calculation of the Integrated Discrimination Improvement (IDI) statistic.

Results: DiaREM was better at predicting T2D remission compared with ABCD (AUCROC 0.75 and 0.67 , respectively). We found that adding AUC of insulin in response to MMT to DiaREM significantly improved the model's predictive performance (AUCROC 0.83, IDI p-value $=0.0076$ ).

Conclusion: The addition of a clinically assessable biomarker (insulin secretion in response to MMT) to DiaREM could improve prediction of diabetes remission at 1-year following surgery.

\section{O5 - The impact of Bariatric Medical Tourism on an NHS Bariatric service from 2015-2021}

Ms Naiara Fernandez Munoz ${ }_{-}^{1}$, Ms Katarina Burton ${ }^{2}$, Ms Cleverly Fong ${ }^{2}$, Mrs Lorna Antoine, Ms Roxanna Zakeri ${ }^{3}$, Ms Emilane Lacea ${ }^{1}$, Mr Naveed Hossain ${ }^{3}$, Mr Ali Alhamdani ${ }^{3}$, Mr Pratik Sufi ${ }^{3}$, Mr Chetan Parmar $^{3}$

${ }^{1} N M C$, London, United Kingdom, ${ }^{2} B D A$, London, United Kingdom, ${ }^{3} G M C$, London, United Kingdom

The lack of affordable bariatric procedures in the private sector and long waiting lists in the public sector are some of the most frequently voiced reasons by patients choosing to engage in Bariatric Medical Tourism (BMT). We analysed the impact of BMT on our bariatric service.
All patients within our service who had undergone primary bariatric procedures outside the United Kingdom were identified through Multidisciplinary Team meeting records.

From April 2015 to April 2021, 65 patients were referred to our service by their GPs due to bariatric surgery-related complications. Initial procedures were performed in 17 countries outside of the UK, Turkey being the most prevalent. Twenty-two patients underwent gastric-band placement, 11 Roux-en-Y gastric bypass, 20 sleeve gastrectomy, 1 mini gastric bypass, 3 revision/conversion surgery and 8 gastric-balloon placement. Twentytwo of the referrals to our service required revisional procedures at our hospital; 12 gastric-band removals, 8 gastric-balloon removals and 2 conversions to another surgery for symptom resolution. The cost of these revisions in the NHS were between $£ 4,000-7,000$ per surgery.

Responsibility for follow-up and monitoring of BMT patients often falls onto NHS services, resulting in significant additional costs and impacting both primary and secondary care resources required for life-long aftercare.

07 - Anxiety and depression amongst participants of the By-BandSleeve Study and short term impact of surgery

Dr Jonathan Gibbs ${ }^{1}$,

Professor Jane Blazeby ${ }^{1}$, By-Band-Sleeve-Trial-Management-Group ${ }^{1}$, By-Band-Sleeve-Trial-Management-Invesigators ${ }^{1}$

\section{${ }^{1}$ University Of Bristol, Bristol, United Kingdom}

Mental health conditions are common amongst patients selected for bariatric surgery but prospective data about the impact of surgery on mental health are limited. We present Hospital Anxiety and Depression Scale (HADS) data from the NIHR By-Band-Sleeve (BBS) Study.

BBS is a 12-centre RCT that has recruited 1351 patients. The validated HADS questionnaire is completed at randomisation and in follow-up. Scores of $<8$ for anxiety and depression are normal, 8-10 suggestive of illness, and $>11$ indicative. Included were patients who underwent surgery within 6 months of randomisation. We examined, i) the prevalence of anxiety and depression (i.e. scores $>8$ ) in all patients with complete baseline questionnaires, and, ii) changes in anxiety and depression from baseline to 12 months in patients with complete data ( $\chi 2$ test).

Baseline prevalence of anxiety and depression was $46.2 \%$ (n 330/716) and $48.2 \%$ (n 343/712) respectively. Paired data for 503 individuals with complete HADS-A and 498 individuals with complete HADS-D showed statistically significant reductions in anxiety $(-9.5 \%)$ and depression ($22.3 \%)$ disorders $(\mathrm{p}<0.001)$.

Patients eligible for bariatric surgery commonly have serious problems with mental health. Improvements are seen in the short-term following surgery. Long-term follow up is needed to understand if benefits are maintained.

O8 - The first COVID-19 'lockdown' period and weight-loss surgery: how did patients cope?

Miss Maureen Boyle_, Miss Aoife McMahon, Miss Claire Lee

${ }^{1}$ South Tyneside and Sunderland NHS Foundation Trust, Sunderland, United Kingdom

Introduction: Owing to the 2020 Covid-19 pandemic, telephone reviews replaced face to face appointments. This study aimed to evaluate the impact of this service change on patients' experiences post-operatively, focusing on diet and lifestyle.

Methods: A bespoke, anonymised 18 point postal questionnaire was sent to all patients $(n=307)$ who had received a post-operative dietetic telephone review between March and July 2020. No identifiable information was collected. 
Results: The questionnaire elicited a $29 \%$ response rate $(n=91)$. The majority of respondents had undergone surgery 6-24 months prior to telephone review. $83 \%(n=76)$ were female. $44 \%(n=40)$ felt their motivation to change diet and lifestyle had not been affected, however $61.5 \%(\mathrm{n}=56)$ reported increased snacking, with $36.3 \%(\mathrm{n}=33)$ consuming more high fat/sugary foods.50.5\% $(n=46)$ reported decreased physical activity. $52.7 \%(n=48)$ stated worsening mental health during 'lockdown' and $50.5 \%(n=46)$ spend increased time thinking about their weight/shape. Overall, alcohol intake increased, with the number of patients drinking 3-7 days/week increasing- 6 fold

Conclusion: The majority of patients felt that 'lockdown' negatively affected their post-operative experiences. Despite positive comments on telephone reviews, the majority of patients preferred face to face appointments.

O9 - Introduction to a digital clinical psychology triage for patients living with complex obesity Dr Lisa Debrou ${ }^{1}$, Ms Rosemary Huntriss ${ }_{-}^{1}$, Mrs Lucy Diamond ${ }^{1}$, Ms Lucy
Jones $^{1}$

${ }^{1}$ Oviva, London, United Kingdom

Introduction: There is a strong relationship between obesity and psychological comorbidity including serious mental illness (National Obesity Observatory, 2011). Psychological support is a key part of a multidisciplinary approach and can be offered digitally allowing scalability and accessibility. Patients benefit from different types of psychological support according to clinical need.

Methods: Using clinical expertise, user feedback and functions of a digital smartphone application, five levels of triage were designed to offer a stepped-care approach depending on need and preference, to be in line with current clinical psychology practices.

Results: Upon referral into a 12-month tier 3 weight management programme, patients are triaged according to their questions and scores relating to psychological morbidity, emotional eating, trauma history, risk of harm to self and binge eating. Based on their score and preference, patients are triaged into one of five levels of care: (1) no intervention; (ii) guided self-help; (iii) app coaching; (iv) telephone/video call with psychological wellbeing practitioner; or (v) clinical psychologist.

Conclusion: Evaluation of impact will be through standardised measures (e.g. PHQ9, BEDS-7 and EQ5D) body metrics (BMI, weight) and programme attendance rates. Expected outcomes are improved programme retention, greater quality of life and improved mood and binge eating scores.

O11 - The impact of COVID-19 on total body weight loss in the first 12-months post bariatric surgery

J Porter $^{1,2,3}$, H Kingsett ${ }^{1,2,3}$, Dr A Brown ${ }^{1,2,3}$, Dr A Pucci ${ }^{1,2}$, Mr M Elkalaawy $^{1}$, Mr A Adamo ${ }^{1}, \mathrm{Mr} \mathrm{M}$ Adamo $^{1}$, Mr A Jenkinson ${ }^{1}, \mathrm{Mr} \mathrm{H}$ Markakis $^{1}$, O Fehilly ${ }^{1}$, K Carr-Rose ${ }^{1}$, H Evett ${ }^{1}$, N Hamid ${ }^{1}$, A Tshiala ${ }^{1}$, Professor R.L. Batterham ${ }^{1,2,3}$

${ }^{1}$ Bariatric Centre for Weight Management and Metabolic Surgery, University College London Hospital NHS Trust, London, UK, ${ }^{2}$ Centre for Obesity Research, University College London, London, UK, ${ }^{3}$ National Institute of Health Research, UCLH Biomedical Research Centre, , UK

Background: The COVID-19 pandemic led to staff re-deployment, cancellation of face-to-face post-surgical clinics and switching to virtual clinics. These changes impacted how bariatric services supported their post-operative patients with potential advise effects on weight loss outcomes. This study aimed to investigate whether
COVID-19 impacted on total body weight loss (TBWL) in the first 12-months post-surgery.

Methods: A retrospective case-control study of 164 patients (mean age 47 \pm 1 years, females [75.5\%], $62.2 \%$ White, mean BMI $\left.46.4 \pm 7.8 \mathrm{~kg} / \mathrm{m}^{2}\right)$ who had surgery between October 2019-March 2020 (post-COVID, n=86) and October 2018-March 2019 (pre-COVID, n=77) was conducted.

Results: Mean TBWL was $23.1 \pm 9.6 \%$ from surgery to 12 -months $(\mathrm{p}<0.001)$, with no difference found between pre- and post-COVID ( $22.8 .3 \pm 9.8 \%$ vs. $23.4 \pm 9.4 \%, \mathrm{p}=0.83$, respectively). No differences were found in baseline demographics. There was a significant difference in the number of appointments $(4$ [IQR 3,5] vs $2[$ IQR 2,2]<0.001) and delivery method (face-to-face vs telephone, $\mathrm{p}<0.001$ ) between pre- and postCOVID. The number of appointments and method did not impact on TBWL ( $\mathrm{p}=0.24$ and 0.34 ).

Conclusion: Despite concerns COVID-19 would negatively impact on weight loss, our data suggests TBWL at 12-months was not impacted. Interestingly, the number of contacts and changing to telephone contact did not impact upon initial weight loss outcome.

013 - Safety and Efficacy of a One Night Stay protocol for patients undergoing elective bariatric surgery at Tertiary Hospital during the COVID-19 pandemic in the United kingdom.s

Dr Shubra Kochar_, Dr Mouhammed Farah, Mr Kamal Mahawar

\section{${ }^{1}$ 1, Sunderland Royal Hospital, United Kingdom}

Introduction: We have recently amended our bariatric protocol from 2-3 nights hospital stay before the pandemic to a planned one-night hospital stay since July 2020 . The purpose of this study was to evaluate the safety and efficacy of the new protocol.

Methods: We conducted a retrospective comparative study of all patients who underwent bariatric surgery at our bariatric unit from July to September 2019 (Cohort A) and compared it to the cohort during the Pandemic from July to September 2020 (Cohort B).

Results: Sleeve Gastrectomy, OAGB, and RYGB constituted $16 \%$, $47.5 \%, 35 \%$ of all procedures in Cohort $\mathrm{A}$ and $17 \%, 43.3 \%, 36 \%$ in Cohort $\mathrm{B}$ respectively. The mean and median peri-operative stay for Cohort B during the pandemic were 1.35 and 1.0 day, as compared to 2.5 and 2.0 days for Cohort A. The 30day mortality, reoperation, morbidity, and readmission in Cohort A were $0,1.3 \%, 1.3 \%, 2.6 \%$ respectively and for Cohort B were $0,0,5.7 \%, 7.5 \%$ respectively.

Conclusion: Our new one night stay bariatric protocol implemented during the COVID-19 pandemic has reduced the peri operative hospital stay. However, it may have increased 30-day minor morbidity and readmissions.

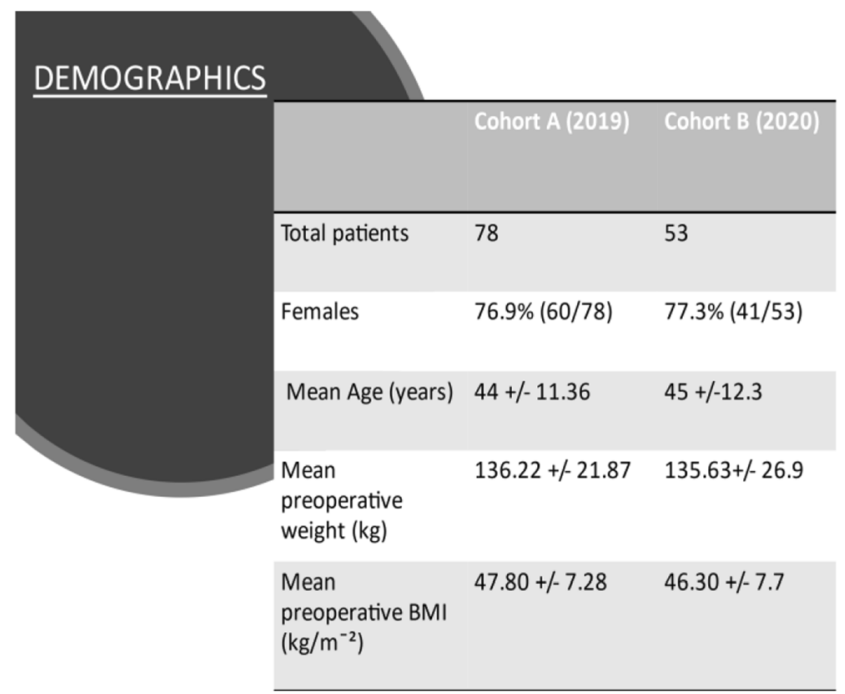


O15 - Management of Laryngotracheal Stenosis in Obesity; Is this another co-morbidity that can be improved with weight loss following Bariatric Surgery?

Miss Anuja Mitra ${ }^{1,2}$, Mr Matyas Fehervari ${ }^{1,2}$, Dr Shivali Patel ${ }^{2}$, Dr Rebecca Towning ${ }^{2}$, Dr Atika Sabharwal ${ }^{2}$, Mr Chadwan Al Yaghchi ${ }^{3}$, Mr Guri Sandhu ${ }^{3}$, Dr Kevin Haire ${ }^{2}$, Mr Evangelos Efthimiou ${ }^{1,2}$

${ }^{1}$ Department of Surgery \& Cancer, Imperial College London, London, United Kingdom, ${ }^{2}$ Department of Bariatric \& Metabolic Surgery, Chelsea \& Westminster Hospital, London, United Kingdom, ${ }^{3}$ Charing Cross ENT Department, London, United Kingdom

Introduction: The prevalence of laryngotracheal stenosis (LTS) is greater in obese patients. Management includes serial endoscopic airway interventions and definitive airway reconstruction which is often not feasible in this population due to significant anaesthetic and surgical risks.

No studies have investigated whether bariatric surgery (BS) is an effective treatment modality for LTS.

Methods: Data was prospectively collected across two tertiary centres of LTS patients with co- existing BMI of $>35 \mathrm{~kg} / \mathrm{m} 2$ between 2014- 2020. LTS patients were managed conservatively or underwent BS (Roux-en-Y gastric bypass or sleeve gastrectomy) and followed for 60 months.

Results: Eleven LTS patients with mean BMI of $43 \mathrm{~kg} / \mathrm{m} 2$ (37-45) were included; 6 underwent BS and 5 were controls. Total weight loss after BS was $19.4 \%(14-24 \%)$ at 12 months compared to $2.3 \%$ (1$3 \%)$ in controls. Significantly fewer endoscopic interventions $(\mathrm{p}=0.002)$ and dilatations $(\mathrm{p}=0.004)$ occurred in the BS group. No patients were suitable for airway surgery before BS however $50 \%$ underwent reconstruction after. LTS patients who had BS and reconstruction required less subsequent interventions in comparison to LTS controls (Figure 1).

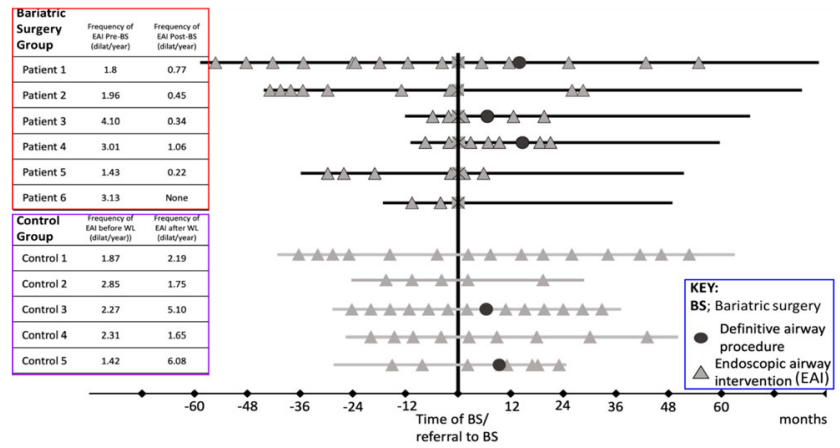

Figure 1: The Frequency of Endoscopic and Definitive Airway Interventions in Laryngotracheal Stenosis patients before and after Bariatric surgery versus Controls

Conclusion: Bariatric surgery is an effective management option for LTS and facilitates definitive airway reconstruction. LTS may be an indication for BS following multidisciplinary assessment.

016 - Gastro-oesophageal reflux disease and oesophagitis following sleeve gastrectomy and gastric bypass.

Ms Tanya Bharat Rajwanshi ${ }^{1}$, Mr Colm J O'Boyle ${ }^{2}$, Mr Hayder Shabana ${ }^{2}$, Ms Roisin Daly ${ }^{2}$, Mr Niall Reddy ${ }^{2}$

${ }^{1}$ University College Cork, Cork City, Ireland, ${ }^{2}$ Bon Secours Hospital, Cork City, Ireland
Introduction: Routine diagnostic endoscopy in either a pre-operative or post-operative bariatric surgical setting is controversial.

Objectives: To evaluate the utility of routine gastroscopy performed preand post- bariatric surgical intervention

Methods:Between June 2008 and June 2018, 260 patients undergoing primary bariatric surgery, under the care of a single Consultant Surgeon, underwent routine pre-operative and post-operative endoscopy.

Results $78 \%$ (204) were female. The mean (SD) age was 46(11) years. The mean preoperative BMI was $49(7) \mathrm{kg} / \mathrm{m} 2$. 73\%(191) underwent laparoscopic gastric bypass (LRYGB) and 26\%(69) underwent sleeve gastrectomy (LSG). The median (range) time to post-operative endoscopy was 26(12-91) months. The median BMI on follow-up was 33(11) kg/m2.

Endoscopic findings of oesophagitis resulted in 11(14\%) patients changing preference from LSG to LRYGB pre-operatively. As a consequence oesophagitis was present in a greater number of LRYGB patients preoperatively $(38(20 \%)$ vs $9(13 \%), \mathrm{p}<0.0001)$. However, post-operatively the incidence of oesophagitis was significantly lower for LRYGB $(5(2.6 \%)$ vs $12(17 \%), \mathrm{p}<0.001)$.

By the time of post-operative endoscopy there was a greater reduction in the number of patients taking medication for GORD in the LRYGB group $(49 \%(50 / 103)$ vs $9 \%(3 / 31), \mathrm{p}<0.001)$.

LSG was associated with a higher incidence of DE NOVO post-operative GORD requiring medication $(10(14 \%) \mathrm{v} 13(7 \%), \mathrm{p}<0.001)$ and $\mathrm{DE}$ NOVO oesophagitis $(10(14 \%)$ v $3(1.5 \%), \mathrm{p}<0.001)$.

Barrett's oesophagus was present in $10(3.8 \%)$ patients pre-operatively. Post-LRYGB 1(10\%) had resolved, 3(30\%) had improved, 2(20\%) had progressed. The four patients in the LSG group remained stable.

Conclusion: Gastric bypass is associated with resolution of GORD symptomatology and endoscopically diagnosed oesophagitis in the majority of patients. This is not the case for LSG. De Novo GORD requiring medical intervention and oesophagitis occurs in both groups - but is more common following LSG. The behaviour of Barrett's oesophagus is unpredictable. However, there was no new diagnosis of Barrett's on follow-up endoscopy.

017 - Semaglutide $2.4 \mathrm{mg}$ once weekly reduces appetite, reduces energy intake, and improves control of eating in subjects with obesity Rachel Batterham ${ }^{1}$, Astrid Breitschaft ${ }^{3}$, Martin Friedrichsen ${ }^{2}$,

Nigel Morgan ${ }^{2}$, Dorthe Skovgaard ${ }^{2}$, Sayeh Tadayon ${ }^{2}$, Alicja Wizert ${ }^{2}$

${ }^{1}$ University College London, London, United Kingdom, ${ }^{2}$ Novo Nordisk A/S, Søborg, , Denmark, ${ }^{3}$ Parexel International GmbH, Berlin, Germany

Introduction: Semaglutide $2.4 \mathrm{mg}$, a glucagon-like peptide-1 analogue (GLP-1RA) administered subcutaneously once-weekly, is under development for chronic weight management. This trial investigated the effect of semaglutide $2.4 \mathrm{mg}$ on gastric emptying, energy intake, appetite and control of eating $(\mathrm{CoE})$ in people with obesity.

Methods: Adults aged 18-65yrs, BMI $30-45 \mathrm{~kg} / \mathrm{m} 2$ and $\mathrm{HbA} 1 \mathrm{c}<6.5 \%$ were randomised to once-weekly semaglutide $2.4 \mathrm{mg}$ or placebo. Gastric emptying (paracetamol absorption), postprandial appetite (visual analogue scales), ad libitum energy intake and $\mathrm{CoE}$ (Control of Eating Questionnaire [CoEQ]) were assessed at baseline and week 20.

Results: 72 subjects were randomised (44 males, mean age $42.8 \mathrm{yrs}$, BMI $34.4 \mathrm{~kg} / \mathrm{m} 2)$. Paracetamol AUC0-5h,para was modestly increased $(8 \%$ $[\mathrm{p}<0.01])$ with semaglutide $2.4 \mathrm{mg}$. No effect was seen on AUC01 h,para,Cmax,para or tmax,para. Significant reductions in energy intake during ad libitum lunch (35\%; 1,736 vs 2,676kJ [ETD, 940kJ; $p<0.0001]$ ), appetite ( $=0.001$; Figure) were observed with semaglutide $2.4 \mathrm{mg}$ vs placebo. Overall, CoEQ indicated fewer and weaker food cravings and improved $\mathrm{CoE}$ with semaglutide $2.4 \mathrm{mg}$ vs placebo $(\mathrm{p}<0.02)$. No new safety signals were observed. 
Conclusion: In people with obesity, semaglutide $2.4 \mathrm{mg}$ suppressed appetite, reduced the frequency and strength of food cravings and reduced ad libitum energy intake. There was no clinically relevant effect on gastric emptying with semaglutide $2.4 \mathrm{mg}$.

Clinical Trial Registration Number: NCT03842202

Supported by: Novo Nordisk

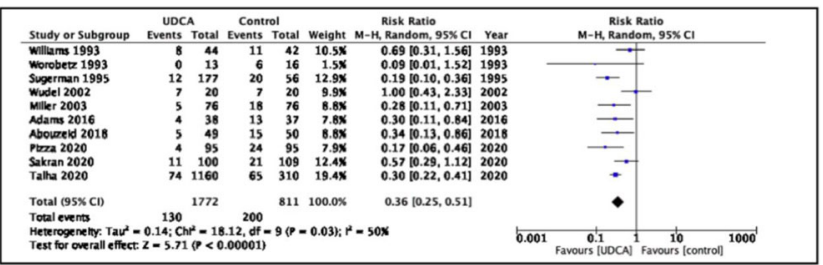

018 - Bariatric surgery restores dysregulated hepatic lipid metabolism and promotes regression across the spectrum of NAFLD

Mr Niall Dempster ${ }^{1,2}, \mathrm{Mr}_{\text {Richard Gillies }}^{2}$, Mr Bruno Sgromo ${ }^{2}$, Dr Garry $\mathrm{Tan}^{1}$, Prof Leanne Hodson ${ }^{1}$, Prof Jeremy Tomlinson ${ }^{1}$

${ }^{1}$ Oxford Centre for Diabetes, Endocrinology and Metabolism, University of Oxford, Oxford, United Kingdom, ${ }^{2}$ Department of General Surgery, Oxford University Hospitals NHS Foundation Trust, Oxford, United Kingdom

Introduction: Non-alcoholic fatty liver disease (NAFLD) is characterised by dysregulated lipid metabolism and is a leading cause of liver failure. It is uncertain how bariatric surgery (BS) improves NAFLD, or whether sleeve gastrectomy (SG) and Rouxen-Y gastric bypass (RYGB) have differential effects. Our aims were to develop mechanistic insight into BS's effects on NAFLD and to compare SG and RYGB outcomes.

Methods: 40 non-diabetic females were recruited ( 24 with obesity who were randomised to SG or RYGB and 16 non-obese controls (NOCs)). Research visits occurred before and after preoperative diet, at $20 \%$ weight loss and 1 year postoperatively. NAFLD was assessed using MRI, and comprehensive phenotyping including isotopically-labelled mixed meal tests was performed.

Results: Imaging and biochemical biomarkers of liver steatosis $(\mathrm{p}<0.01)$, inflammation $(\mathrm{p}=0.01)$ and fibrosis $(\mathrm{p}<0.01)$ improved longitudinally. Hepatic de novo lipogenesis products including myristic acid $(\mathrm{p}=0.01)$ decreased, whilst lipid oxidation marker 3-hydroxybutyrate increased $(p<0.01)$. Although postprandial response differed after SG or RYGB, both normalised liver steatosis relative to NOCs $(\mathrm{p}=0.14-0.29)$.

Conclusions: BS was an effective intervention for NAFLD, normalising liver steatosis through suppression of hepatic de novo lipogenesis and increased lipid oxidation. Postprandial response and serum metabolome were distinct after SG or RYGB, but both induced NAFLD regression.

O19 - Increased colonic permeability to oral chromium (51Cr)-EDTA in type two diabetics undergoing Roux-en-Y gastric bypass, compared to a non-operated control group

Mr James O'Brien ${ }^{1}$, Dr Denise Robertson ${ }^{2}$, Prof Bruce Griffin ${ }^{2}$, Prof Timothy Rockall ${ }^{1}$, Mr Chris Pring ${ }^{2}$

${ }^{I}$ MATTU, Royal Surrey Hospital, Guildford, United Kingdom,

${ }^{2}$ University of Surrey, Guildford,, United Kingdom

Introduction: The mechanism of action of bariatric surgery is poorly understood but thought to involve altered gastrointestinal permeability (gut barrier function). We examined the relationship between Roux-enY bypass (RYGB), permeability and diabetes, and hypothesised that permeability in an operated diabetic group would improve compared to non-operated controls.

Methods: Permeability was analysed using urinary Cr51-EDTA, in 15 type two diabetics, at baseline and twelve weeks after RYGB. This was compared to 15 non-operated diabetics of average BMI, matched for age, gender and time with diabetes.

Results: No difference in baseline small intestinal and colonic permeability. Following surgery small intestinal and overall permeability did not change in either group, but in the RYGB group colonic permeability significantly increased $(\mathrm{p}<0.001)$ becoming different to controls $(\mathrm{p}=0.001)$. There was significant reduction in mean BMI $(11.6 \mathrm{~kg} / \mathrm{m} 2)$ with no change in controls, and a significant reduction in mean $\mathrm{HbA} 1 \mathrm{c}(24.6 \mathrm{mmol} / \mathrm{mol})$ compared to controls $(\mathrm{p}<0.001)$.

Conclusion: Despite BMI and diabetes improving, small intestinal permeability post-RYGB did not change. In keeping with findings following sleeve gastrectomy, colonic permeability deteriorated after RYGB.(1) This suggests that altered permeability may not be directly dependent on changes to weight or diabetes, and indicates surgery may have a detrimental effect on colonic permeability.

\section{References}

1 Kellerer T, Brandl B, Büttner J, Lagkouvardos I, Hauner H, Skurk T. Impact of Laparoscopic Sleeve Gastrectomy on Gut Permeability in Morbidly Obese Subjects. Obes Surg. 2019 Jul;29(7):2132-2143. doi: 10.1007/s11695-019-03815-6. PMID: 30903427.

O20 - The impact of ursodeoxycholic acid on gallstone disease after bariatric surgery: a meta-analysis of randomised control trials

Ms Naomi Fearon ${ }^{1,4}$, Dr Emma Kearns ${ }^{2}$, Ms Czara Kennedy ${ }^{3}$, Mr John Conneely ${ }^{4}$, Professor Helen Heneghan ${ }^{2,5}$

${ }^{1}$ RCSI, Dublin, Ireland, ${ }^{2}$ St Vincent's University Hospital, Dublin, Ireland, ${ }^{3}$ Mullingar Regional Hospital, Mullingar, Ireland, ${ }^{4}$ Mater Misericordiae University Hospital, Dublin, Ireland, ${ }^{5}$ University College Dublin, Dublin, Ireland

Purpose: The benefits of ursodeoxycholic acid (UDCA) after bariatric surgery have been described, however it is not currently the standard of care. This study aimed to evaluate the impact of UDCA on gallstone disease after bariatric surgery.

Methods: A meta-analysis of randomised control trials (RCT) was performed. The primary outcome was formation of gallstones. Secondary outcomes included type of operation, time interval to gallstone formation and symptomatology.

Results: Ten randomised control trials including 2583 patients were included, 1772 patients $(68.6 \%)$ receiving UDCA and 811 (31.4\%) receiving placebo. There was a significant reduction in gallstone formation in patients who received UDCA post-operatively (RR 0.36 , 95\% CI 0.22 , $0.41, p=<0.00001$ ) shown in Figure 1. Subgroup outcomes are presented in Table 1 . A dose of $\leq 600 \mathrm{mg}$ day had a significantly reduced risk of gallstone formation compared to controls (RR 0.35; 95\% CI 0.24, 0.53; $\mathrm{P}<0.001$ ), this was not significant in $>600 \mathrm{mg} /$ day $(\mathrm{RR} 0.30 ; 95 \% \mathrm{CI}$, $0.09,1.01, \mathrm{p}=0.05$ ).

Conclusion:

UDCA reduces the risk of asymptomatic and symptomatic gallstones after bariatric surgery. A lower dose is associated with improved compliance and better outcomes. UDCA should be considered part of a standard postoperative care bundle. 


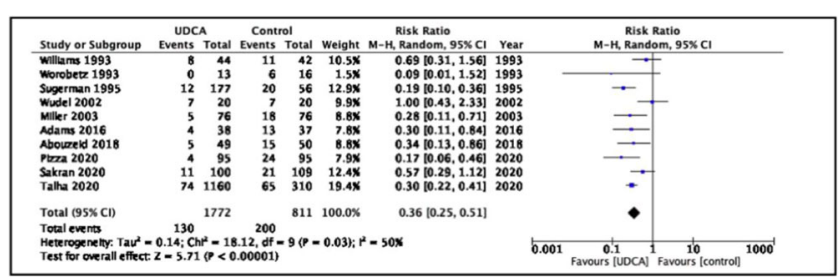

Figure 1.

\begin{tabular}{|l|c|c|c|c|}
\hline Outcome or subgroup & Studies & $\mathbf{n}$ & $\begin{array}{c}\text { Effect estimate RR } \\
{[95 \% \text { Cl] }}\end{array}$ & Heterogeneity I $^{\mathbf{2}}(\%)$ \\
\hline $\begin{array}{l}\text { Gallstone formation in relation to dose of } \\
\text { UDCA }\end{array}$ & & & & \\
\hline Low dose $5600 \mathrm{mg} /$ day & $\mathbf{3}$ & 2405 & $0.35[0.24,0.49]$ & 44 \\
\hline High dose >600mg/day & 3 & 234 & $0.30[0.09,1.01]$ & 64 \\
\hline $\begin{array}{l}\text { Gallstone formation in relation to type of } \\
\text { surgery }\end{array}$ & & & & \\
\hline Sleeve gastrectomy & 3 & 201 & $0.32[0.18,0.58]$ & 0 \\
\hline Vertical banded gastroplasty & 2 & 115 & $0.38[0.06,2.59]$ & 52 \\
\hline Gastric bypass & 4 & 496 & $0.37[0.16,0.85]$ & 83 \\
\hline Time interval to gallstone formation & & & & \\
\hline 3months & 3 & 305 & $0.31[0.07,1.46]$ & 43 \\
\hline 6months & 5 & 806 & $0.28[0.16,0.48]$ & 38 \\
\hline 12months & 6 & 779 & $0.33[0.20,0.55]$ & 51 \\
\hline $\begin{array}{l}\text { Symptoms in patients who formed } \\
\text { gallstones }\end{array}$ & & & & \\
\hline Symptomatic & 3 & 1686 & $0.24[0.15,0.40]$ & 0 \\
\hline Asymptomatic & 3 & 1686 & $0.32[0.22,0.47]$ & 0 \\
\hline
\end{tabular}

Table 1.

O21 - Duration in a Tier 3 does not improve weight loss or progression to surgery

Dr Anil Rai ${ }^{1}$, Mr Chanpreet Arhi ${ }^{1}$, Ms Yvonne McKeown ${ }^{1}$, Mr Aruna Munasinghe $^{1}$, Mr Farhan Rashid ${ }^{1}$, Mr Md Tanveer Adil ${ }^{1}$, Dr Tariq Rehman $^{1}$, Mr Periyathambi Jambulingam ${ }^{1}$, Mr Vigyan Jain ${ }^{1}$, Mr Omer Al-Taan ${ }^{1}$, Mr Douglas Whitelaw ${ }^{1}$

${ }^{1}$ Luton And Dunstable Hospital, Luton, United Kingdom
Introduction: Commissioners expect patients to complete tier 3 before being considered for tier 4 as there is a presumption a percentage total weight loss (\%TWL) proves readiness and engagement. This study hypothesises that the duration in Tier 3 does not correlate with an improvement in \% TWL nor progression to tier 4 .

Method: Retrospective analysis of referrals to our tier 3 service between June 2018 to February 2020. Univariate ANOVA compared the mean \% TWL according to time in tier $3(<6 \mathrm{~m}, 6-12 \mathrm{~m}$ and $>12 \mathrm{~m})$ while considering age, gender, initial BMI and trial of low calorie $\operatorname{diet}(\mathrm{LCD})$. Logistic regression determined the OR of progression to tier 4 .

Results: 92,93 and 42 patients spent $<6 \mathrm{~m}, 6-12 \mathrm{~m}$ and $>12 \mathrm{~m}$ respectively in Tier 3 (overall mean BMI $51.0 \mathrm{~kg} / \mathrm{m} 2$, mean age 44.3). 107 progressed to tier $4.34 .4 \%, 34.4 \%$ and $11.5 \%$ patients achieved $0-5,5-10$ and $>10$ $\%$ TWL respectively, while the remaining $19.8 \%$ gained weight.

For BMI $>50 \mathrm{~kg} / \mathrm{m} 2$, average $\%$ TWL was $5.5 \%$, compared with $2.6 \%(\mathrm{p}=0.06), 2.9 \%(\mathrm{p}=0.01)$ and $2.2 \%(\mathrm{p}=0.02)$ for $30-39.9,40-44.9$ and $45-50 \mathrm{~kg} / \mathrm{m} 2$ respectively. LCD significantly improved \% TWL (6.0 vs $2.4 \mathrm{p}<0.01)$. However \%TWL did not differ according to the time periods considered $(4.2 \%, 4.1 \%$ and $3.9 \%$ respectively, $\mathrm{p}=0.91)$.

Although higher weight loss during tier 3 significantly increased the likelihood of progression (OR1.58 95\%CI 1.39-1.81 p<0.01), spending 6-12 months significantly reduced the OR of progression compared with those $<6 \mathrm{~m}$ (OR 0.28 95\% CI 0.09-0.92 p=0.04).

Conclusion:In their current format tier 3 services are acting as barriers for MBS. Instead their aim should be to prepare patients about the expected changes after MBS.

Publisher's Note Springer Nature remains neutral with regard to jurisdictional claims in published maps and institutional affiliations. 